\title{
The interplay between miR156/SPL13 and DFR/WD40-1 regulate drought tolerance in alfalfa
}

\author{
Biruk A. Feyissa ${ }^{1,2}$, Muhammad Arshad ${ }^{1,3}$, Margaret Y. Gruber ${ }^{4}$, Susanne E. Kohalmi ${ }^{2}$ and Abdelali Hannoufa ${ }^{1,2^{*}}$ (D)
}

\begin{abstract}
Background: Developing Medicago sativa L. (alfalfa) cultivars tolerant to drought is critical for the crop's sustainable production. miR156 regulates various plant biological functions by silencing SQUAMOSA-PROMOTER BINDING PROTEINLIKE (SPL) transcription factors.

Results: To understand the mechanism of miR156-modulated drought stress tolerance in alfalfa we used genotypes with altered expression levels of miR156, miR156-regulated SPL13, and DIHYDROFLAVONOL-4-REDUCTASE (DFR) regulating WD40-1. Previously we reported the involvement of miR156 in drought tolerance, but the mechanism and downstream genes involved in this process were not fully studied. Here we illustrate the interplay between miR156/ SPL13 and WD40-1/DFR to regulate drought stress by coordinating gene expression with metabolite and physiological strategies. Low to moderate levels of miR156 overexpression suppressed SPL13 and increased WD40-1 to fine-tune DFR expression for enhanced anthocyanin biosynthesis. This, in combination with other accumulated stress mitigating metabolites and physiological responses, improved drought tolerance. We also demonstrated that SPL13 binds in vivo to the DFR promoter to regulate its expression.
\end{abstract}

Conclusions: Taken together, our results reveal that moderate relative miR156 transcript levels are sufficient to enhance drought resilience in alfalfa by silencing SPL13 and increasing WD40-1 expression, whereas higher miR156 overexpression results in drought susceptibility.

Keywords: Alfalfa, Drought, microRNA, miR156, SQUAMOSA-PROMOTER BINDING PROTEIN-LIKE13, WD40-1

\section{Background}

The effects of climate change are expected to result in frequent and extreme weather events causing major damage to crop production $[1,2]$. Plants respond to these changes (abiotic stress) by developing different resilience mechanisms at the phenotypic, physiological and molecular levels [3]. To improve plant response, microRNAs provide an opportunity to mend alfalfa traits [4].

MicroRNAs are small RNAs of approximately 16-26 nucleotides in length that regulate gene expression at the posttranscriptional level in a sequence-specific manner [5]. Of the hundreds of microRNAs [6], microRNA156

\footnotetext{
* Correspondence: Abdelali.hannoufa@canada.ca

${ }^{1}$ Agriculture and Agri-Food Canada, 1391 Sandford Street, London, Ontario N5V 4T3, Canada

${ }^{2}$ Department of Biology, University of Western Ontario, 1151 Richmond

Street, London, Ontario N6A4B7, Canada

Full list of author information is available at the end of the article
}

(miR156) is highly conserved in plants, where it functions by down-regulating a group of SQUAMOSAPROMOTER BINDING PROTEIN-LIKE (SPL) transcription factors [7-9]. There are at least eight members (a to h) of miR156 in Arabidopsis thaliana, with $g$ and h unique to this species. A smaller number of miR156 members (a to f) have been discovered in other plant species, including Medicago truncatula [10]. SPLs regulate a network of downstream genes affecting plant development and physiology by binding to gene promoters at a consensus DNA sequence NNGTACR (where $\mathrm{N}=$ any nucleotide, $\mathrm{R}=\mathrm{A}$ or G) known as the SPL Binding Domain (SBD) [11-14]. In Arabidopsis, miR156 regulates 10 out of 16 SPLs, affecting various aspects of plant growth and development [15], whereas in alfalfa, miR156 regulates at least seven SPLs (SPL2,3,4,6,9,12 and 13) [8]. Despite the conservation of miR156 among plant species, some of its regulation outputs are species-specific $[9,13,16]$. We previously showed

(c) The Author(s). 2019 Open Access This article is distributed under the terms of the Creative Commons Attribution 4.0 International License (http://creativecommons.org/licenses/by/4.0/), which permits unrestricted use, distribution, and 
that overexpression of miR156 in alfalfa delays flowering time, enhances root nodulation, and improves vegetative and root growth $[7,13]$. Many of these traits are associated with abiotic stress tolerance $[17,18]$. Moreover, overexpression of miR156d was shown to improve alfalfa's tolerance to heat [19], salinity [20] and drought stress [21]. miR156-mediated silencing of SPL2, SPL9 and SPL11 improved heat, salt and drought stress resilience in Arabidopsis and rice [22, 23]. Arabidopsis mutants with increased miR156 expression silenced SPL9, and enhanced expression of DIHYDROFLAVONOL-4REDUCTASE (DFR) and PRODUCTION OF ANTHOCYANIN PIGMENT 1 (PAP1), which resulted in increased anthocyanin accumulation and improved stress tolerance [22]. The enhancement of anthocyanins and proanthocanidins is regulated by transcription factors such as WD40, MYB and bHLH $[24,25]$. These secondary metabolites scavenge free radicals during plant abiotic stress [26-28] and function in a coordinated manner with transient stress-related primary metabolites such as proline, galactinol, raffinose and gammaaminobutyric-acid (GABA) to alleviate stress symptoms $[26,29]$.

We recently reported that drought stress enhances miR156 expression to improve alfalfa's resilience to this stress by increasing leaf gas exchange and abscisic acid (ABA), while reducing water loss [21]. Despite these findings, our understanding of how the miR156/SPL network regulates downstream genes such as DFR and WD40-1 to affect stress tolerance in alfalfa is unknown, especially as it relates to drought stress and secondary metabolism. In this study, we investigated the mechanism of how miR156 regulates drought stress response in alfalfa. To that end, we analyzed miR156 over-expressors, SPL13-silenced genotypes, WD40-1 over-expressors and WD40-1 RNAi silenced genotypes at the metabolomic, transcriptomic and physiological levels. Moreover, we investigated the binding of SPL13 to the DFR promoter to regulate flavonoid biosynthesis. The findings from this report would be useful to understand the mechanisms deployed by miR156 in regulating drought stress and could be used as a tool in marker-assisted breeding to improve alfalfa and potentially other crops.

\section{Results}

Enhanced miR156 expression improves drought tolerance by altering root architecture and water holding capacity

To determine drought stress regulation by miR156, we used one-month-old miR156OE alfalfa plants with low $(\mathrm{A} 8 \mathrm{a}=0.5)$, moderate $(\mathrm{A} 8=1.5)$ and higher $(\mathrm{A} 11=2.5)$ relative miR156 expression levels than the empty vector (EV) [13] grown under drought and well-watered conditions. Root weight, root length, stem basal width and fresh root-to-shoot weight ratios were affected by drought stress depending on the genotype (Fig. 1, Additional file 2: Table S5.1). Relative to EV, A8a had significantly longer roots and increased root biomass (Fig. 1a), with increases of root length up to 1.8-fold (Fig. 1b) and 1.7-fold in root weight (Fig. 1c). The increment of root biomass in A8a was the result of longer roots rather than short and thicker roots (Fig. 1b,c). To understand if the improved root architecture affected plant water potential, we measured leaf water potential [30] and changes in the lower stem diameter before and after drought [31-33]. MiR156OE genotypes, A8a and A8, maintained a higher leaf water potential (Fig. 1f) and also either maintained or increased basal stem diameter (Fig. 1d) while EV plants showed a reduction over the 2 weeks of stress. The unchanged basal stem diameter was accompanied by an increase in root/shoot biomass ratio in A8a and A8 (Fig. 1e).

miR156 overexpression affects photosynthesis parameters Since drought stress negatively affects photosynthesis parameters [34], we investigated this effect in miR156OE and EV plants. Accordingly, photosystem II (PS II) chlorophyll fluorescence, $\mathrm{Fv} / \mathrm{Fm}$ ratio, was measured. Fv/Fm was significantly affected by genotype, drought exposure time, and a combination of both (Additional file 2: Table S5.1). MiR156OE plants maintained higher levels of $\mathrm{Fv} / \mathrm{Fm}$ ratio (0.75) at later stages (day 11 and 14) comparable to unstressed plants while EV plants showed a gradual reduction to 0.69 after 14 days of drought (Fig. 1i). Furthermore, photosynthesis assimilation rate was significantly affected by genotype and the duration of drought exposure (Additional file 2: Table S5.1). Our data revealed that during drought stress the photosynthetic assimilation rate was higher in A8, gradually decreased in A8a, and further decreased in A11except on day 14 when it was greater than in EV (Fig. 1j).

Moreover, the maximum rate of rubisco carboxylase activity $V c \max$ was maintained at a relatively higher level in A8a and A8 plants while a comparably significant reduction (64-75\%) was observed in EV and A11 plants during drought stress (Fig. 1g). In line with this, maximum photosynthetic electron transport rate Jmax was also maintained at higher levels in A8a and A8 during drought stress while it was reduced (64\%) in EV and A11 plants (Fig. 1h).

miR156OE plants accumulate anthocyanin and other stress-related secondary metabolites under drought Using more than 4000 metabolite features, a Principal Component Analysis (PCA) plot of LCMS-based metabolite profiles depicted a distinct difference between drought-treated EV and miR156OE stem tissues (Fig. 2a). These metabolite features are spectral data generated from metabolites [35, 36]. Principal component-1 (PC-1) contributed $32.7 \%$ of the variance and clearly separated 
a

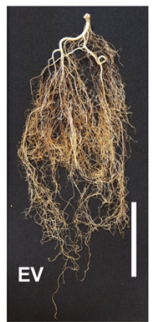

b

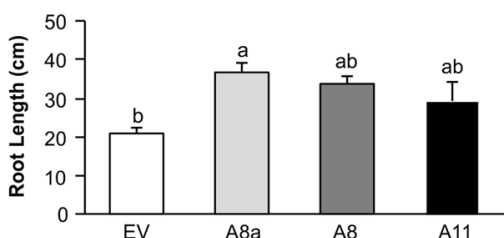

C

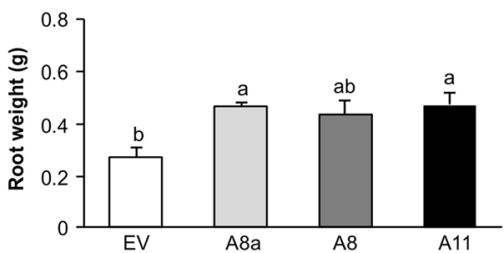

d
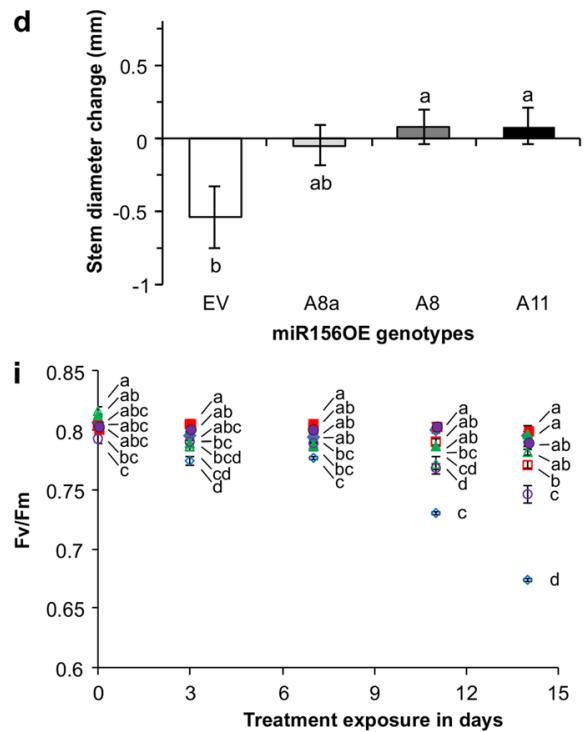
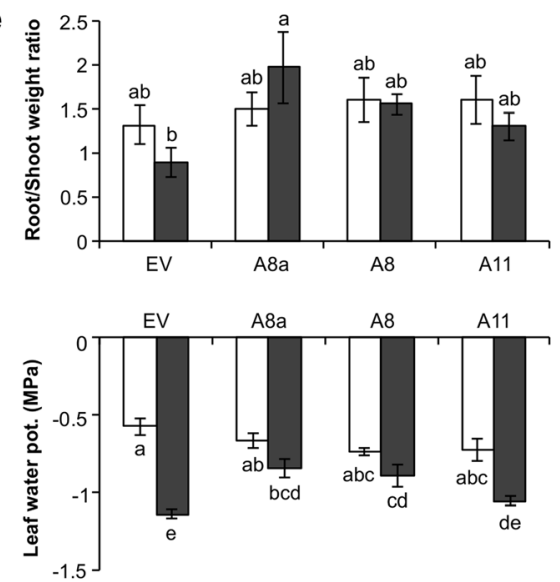

g
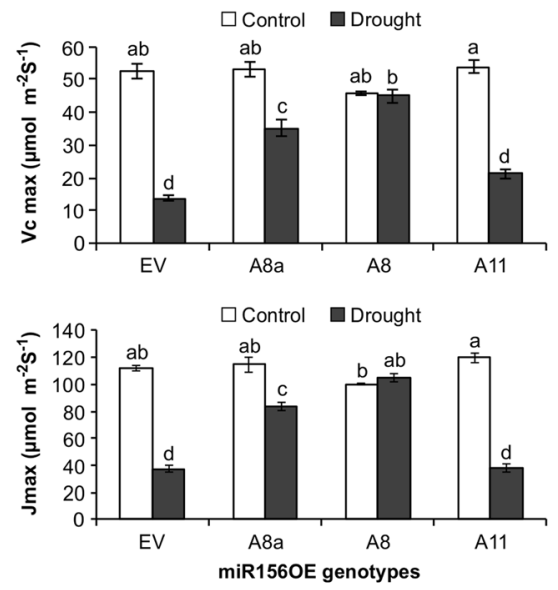

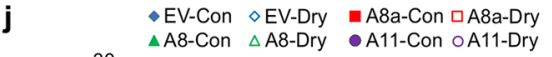

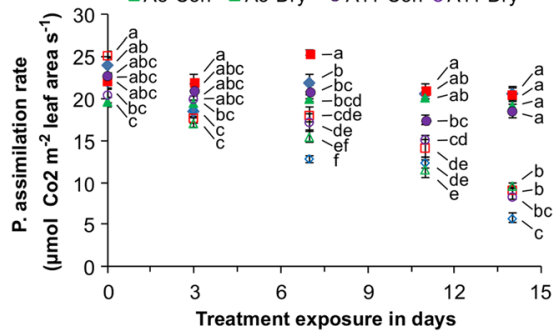

Fig. 1 Effects of miR156 overexpression on drought tolerance and physiological responses in alfalfa. a Roots of EV and miR156OE plants under drought stress; b root length; c root weight; $\mathbf{d}$ stem basal diameter change under drought; e root/shoot biomass ratio; $\mathbf{f}$ leaf water potential; $\mathbf{g}$ Vcmax, maximum rate of rubisco carboxylase activity; $\mathbf{h}$ Jmax, maximum rate of photosynthetic electron transport; i dark adapted chlorophyll florescence, FV/Fm, and $\mathbf{j}$ photosynthetic assimilation rate in well-watered (control) and drought stressed plants. Values are sample means \pm SE, $n=4$ individual plants except in ' $\mathbf{d}$ ', 'e', 'f', 'i', 'j' where $n=5$. ANOVA $p$ values are provided in Additional file 2: Table S5.1. Significant difference in Post hoc Tukey multiple comparisons test is indicated with different letters. Letters in multiple time point data of 'i' and ' $\mathbf{j}$ ' is analyzed separately

EV and miR156OE genotypes stem samples while principal component-2 (PC-2) accounted for $13 \%$ of the variance.

Unlike stem tissues (Fig. 2a), roots possessed a differential metabolite features profile for all genotypes with PC-1 and PC-2 variance of 19.21 and $11.05 \%$, respectively (Fig. 2c). On the other hand, leaves of A8a and EV were metabolically closer (Fig. 2b), whereas the higher miR156 expressor, A11, possessed a different metabolic profile, with PC-1 and PC-2 variance of 18.85 and $12.96 \%$, respectively. Based on their significance level and fold change relative to $\mathrm{EV}$, the numbers of metabolite features common or different in stem, leaf and root of miR156OE genotypes under drought stress are presented in Fig. 2d, e and $\mathrm{f}$, respectively. Figure $2 \mathrm{~d}$ reveals a communal relatively high number of differentially abundant metabolite features (770) between stems of miR156OE and EV plants. The majority $(85.1,81.1$, and $73.4 \%$ for $\mathrm{A} 8 \mathrm{a}, \mathrm{A} 8$, and $\mathrm{A} 11$, 


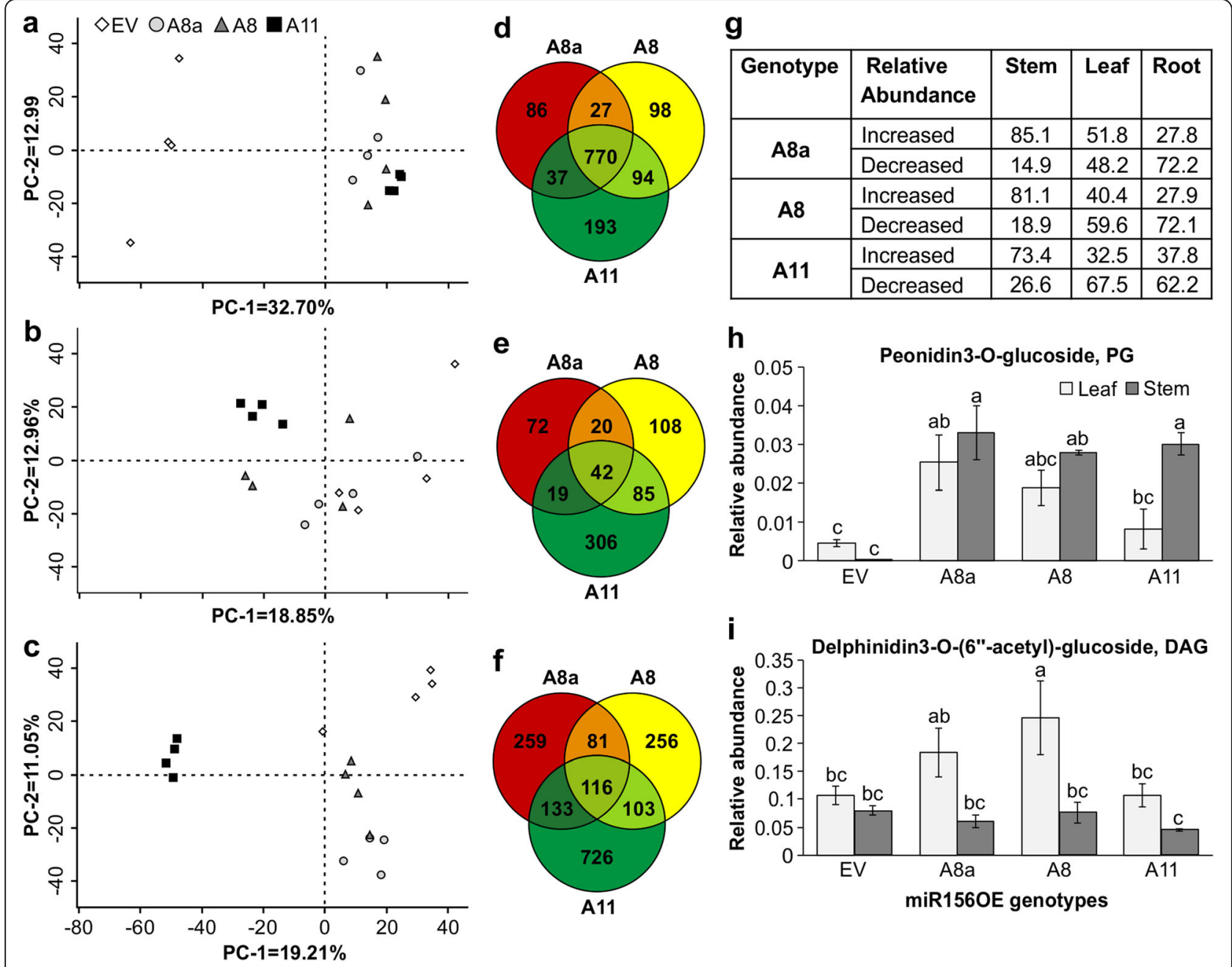

Fig. $\mathbf{2}$ LCMS-based metabolite profiling illustrates distinct profile in miR156OE genotypes during drought stress. a Principal component analysis of metabolite profile in stem, $\mathbf{b}$ leaf, and $\mathbf{c}$ root tissues under drought stress; $\mathbf{d}$ metabolite features that are significantly different at $p<0.01$ from EV plants in tissues of stem, e leaf, and $\mathbf{f}$ root tissues; $\mathbf{g}$ proportion of metabolite features that are significantly increased ( $\geq 1.5$ log 2 fold change) or decreased ( $\leq-1.5 \log 2$ fold change) relative to EV under drought stress; $\mathbf{h}$ relative levels of anthocyanin metabolites of peonidin 3-O-glucoside, PG, and $\mathbf{i}$ delphinidin 3-O-(6"-acetyl)-glucoside, DAG. The relative abundance of metabolites is normalized to an internal standard. Values are sample means $\pm \mathrm{SE}, n=4$ individual plants. ANOVA p values are provided in Additional file 2: Table S5. 4. Significant difference in Post hoc Tukey multiple comparisons test is indicated with different letters

respectively) of the differentially abundant stem metabolites are significantly increased in comparison to EV stem (Fig. 2g). The differential metabolite feature between miR156OE and EV is likely associated with the commonly observed pigmentation of the stem basal internode in miR156OE plants (Additional file 2: Figure S1).

Drought stress induces production of reactive oxygen species (ROS) [37], and plants employ several strategies, including secondary metabolite antioxidants to decrease ROS [38]. Of the many secondary metabolites used by plants as antioxidants, anthocyanins are well documented [39, 40]. Here, levels of anthocyanins such as peonidin 3-O-glucoside (PG) and delphinidin 3-O-
(6" -acetyl)-glucoside (DAG) were significantly affected by genotype and tissue (Additional file 2: Table S5.4). LCMS-based metabolite profiling showed anthocyanins and other ROS scavenging phenolic metabolites were increased mainly in stems of low-to-medium miR156 expressors (A8a and A8), although PG was also increased in A11 (Fig. 2h, i and Additional file 2: Table S2). Acylation of the sugar moiety in anthocyanins increases metabolite stability [41, 42]. It remains to be determined whether such acylation is a factor in leaves of A8 having higher levels of DAG relative to A11 and EV resulting in improved drought tolerance (Fig. 2i). 
Alfalfa plants expressing moderate levels of miR156 accumulate stress-related primary metabolites under drought

Plants coordinate primary and secondary metabolites for tight metabolite regulation and stress response [27, 28, 43]. Hence, we used GCMS for analysis of primary metabolites to determine their levels during drought stress. Results indicated that metabolite levels were governed by tissue and genotype (Additional file 2: Table S5.5). In general, the relative abundance of proteinogenic amino acids was higher in leaf tissues of moderate miR156OE plants, but reduced in highly overexpressing A11 plants (Fig. 3 and Additional file 2: Table S3). With the exception of valine, which showed no significant differences among stem, root and leaf tissues, levels of proteinogenic amino acids were significantly affected by tissue type and a combination of genotype and tissue (Additional file 2: Table S5.5). Alanine, asparagine, glycine and tryptophan showed a relatively higher abundance in leaves of A8 (Fig. 3a). Interestingly, proline, which functions as an osmolyte to maintain plant water potential [26], was significantly increased in root tissues of A8a, comparable in A8 but was reduced in leaf, stem and root tissues of A11 compared to EV plants (Fig. 3b).

Levels of gamma-aminobutyric acid, GABA, a stressresponsive metabolite that mediates carbon to nitrogen balance between glutamate and succinate in the TCA cycle [29], were enhanced in root tissues of A8 and A8a

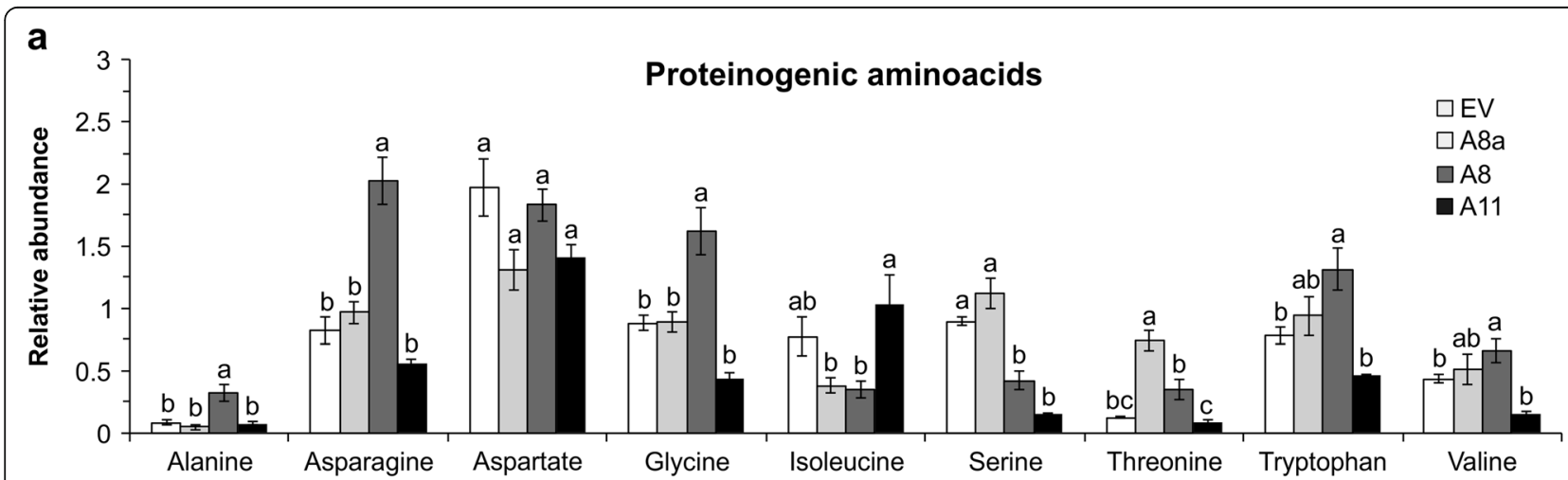

b
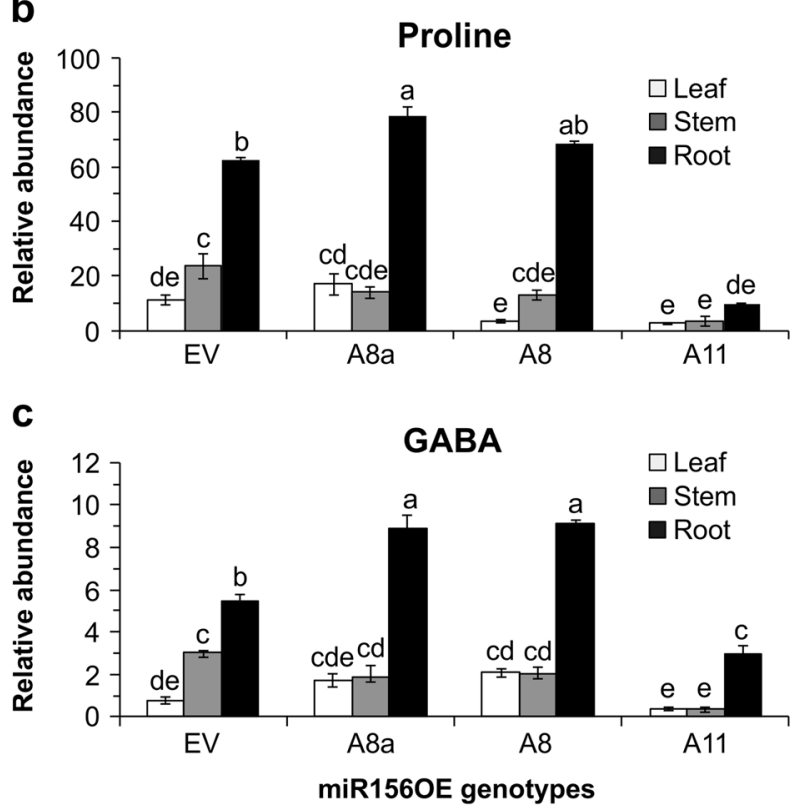

d

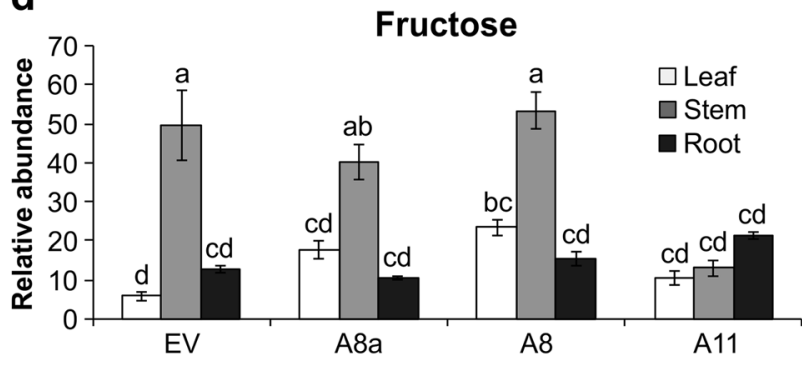

e

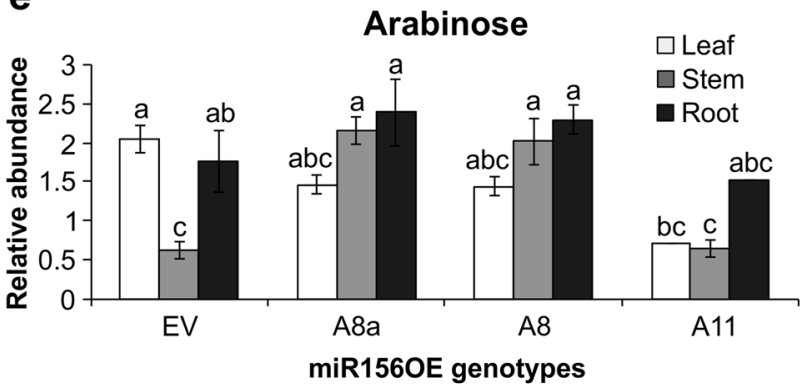

Fig. 3 GCMS-based primary metabolite profiling demonstrates drought stress tolerance strategies by miR156. a Relative levels of proteinogenic amino acids in leaf tissues during drought stress: alanine, asparagine, aspartate, glycine, isoleucine, serine, threonine, tryptophan and valine; $\mathbf{b}$ relative levels of metabolites from the $\gamma$-aminobutyric acid (GABA) shunt in leaf, stem and root tissues of proline, and $\mathbf{c}$ GABA; $\mathbf{d}$ relative levels of sugars from tissues of leaf, stem and root as fructose, and e arabinose under drought stress. Values are sample means \pm SE, $n=4$ individual plants. ANOVA p values are provided in Additional file 2: Table S5.5. Significant difference in Post hoc Tukey multiple comparisons test is indicated with different letters 
(Fig. 3c). The higher miR156 over-expressor, A11, on the other hand, reduced GABA levels in all tissues as compared to EV (Fig. 3c).

An increased level of fructose, one of the main sugar sources for the carbon skeleton of downstream metabolites and a source of energy, was observed in leaf tissues of A8 while its levels were unchanged in stems and roots (Fig. 3d). On the other hand, A11 had variable levels of fructose (Fig. 3d), with levels being reduced in stems but comparable in roots.

Conversion of carbon sources from sugars into the downstream pathways including glycolysis and pentose phosphate pathway (PPP) is of great importance in stress response and tolerance $[44,45]$. Arabinose, an important component of cell wall polysaccharides, PPP, and a major component of glycoproteins and arabinogalactan proteins, had enhanced levels in stems while it was unchanged in leaf and roots of A8a and A8 while reduced in roots of A11 compared to EV (Fig. 3e and Additional file 2: Table S5.5). A complete list of annotated metabolites using GCMS analysis is presented in Additional file 2: Table S3.

\section{Overexpression of miR156 affects expression of photosynthesis and flavonoid genes}

Our physiological and metabolite profiling analysis showed that alfalfa plants overexpressing miR156 at lowto-moderate levels (A8a and A8) have higher anthocyanin levels (Fig. 2h,i) and maintained higher photosynthetic efficiency during drought stress (Fig. 1g-k). We, therefore, investigated if these are regulated at the molecular level by determining relative transcript levels of genes involved in the flavonoid and photosynthetic pathways. Genotype, tissue and their interaction have a significant impact on the transcript levels of flavonoid biosynthesis DFR and MYB112 genes, although MYB112 showed little difference between tissues (Additional file 2: Table S5.6). Accordingly, higher transcript levels of $D F R$ and MYB112 were observed in stem and leaf tissues of at least some miR156OE plants.

DFR, which catalyses the conversion of dihydroflavonol to leucoanthocyanidin, had two- to 15 -fold higher transcription in miR156OE leaf tissues compared to EV (Fig. 4a). DFR transcription was also 25 to 35 -fold higher in miR156OE root samples. MYB112 encodes a transcription factor that regulates flavonoid biosynthesis [46]. Its transcript level was five- to 19 times higher in leaf tissues of miR156OE compared to EV while a four-fold higher expression level was observed in miR156OE stem tissues regardless of genotype (Fig. 4b). A slight increment in the expression level of WD40-1 (1.9-fold), a transcription factor in the phenylpropanoid pathway, was observed in A8 root tissues while it was decreased in stem and leaf tissues (Fig. 4c). Moreover, FLAVONOID
GLUCOSYLTRANSFERASE2 (FGT2), which catalyses the transfer of a glycosyl group onto flavonoids, was significantly increased up to six-fold in leaves of A8a while a 19fold increment was observed in roots (Fig. 4d).

Photosynthesis efficiency related PHOTOSYSTEM I p700 CHLOROPHYLL A APOPROTEIN APS I (PSI) and PHOTOSYSTEM II Q(b) (PSII) transcript levels were affected by genotype and tissue type (Additional file 2: Table S5.6). PSI and PSII transcripts were five and four-fold higher in A8a leaves and roots, respectively (Fig. 4e, f). On the other hand, these two genes were significantly decreased in stems of miR156OE plants (Fig. 4e, f). Stems of miR156OE plants had pigmentation at the basal internode consistent with enhanced anthocyanin accumulation, which interferes with typical green chlorophyll colouration (Additional file 2: Figure S1) [47-49].

\section{SPL13 regulates physiological responses and anthocyanin} accumulation during drought stress in alfalfa

Since miR156 functions in alfalfa by downregulating SPL genes, including SPL13 $[8,13]$, we investigated the effect of drought on the physiological and phenotypic parameters of alfalfa plants having RNAi-silenced SPL13. We previously reported that a green, normal appearing phenotype accompanied enhanced root development in SPL13RNAi genotypes under drought [21]. In the current study, leaf water potential was significantly affected by genotype under drought stress (Additional file 2: Table S5.2). In line with this, SPL13RNAi-5 and SPL13RNAi-6 plants maintained higher midday leaf water potential during drought stress (Fig. 5a). Moreover, photosynthesis efficiency parameters showed that SPL13RNAi-5 and SPL13RNAi-6 with moderate SPL13 silencing [21] maintained a higher $\mathrm{Fv} / \mathrm{Fm}$ ratio of 0.74 (Fig. $5 \mathrm{~b}$ ) after 8 days of drought stress. The level of $\mathrm{Fv} / \mathrm{Fm}$ is significantly affected by genotype, length of drought exposure and a combination of both (Additional file 2: Table S5.2). As a stress tolerance strategy, plants use flavonoids such as anthocyanin to scavenge ROS, and in our study we observed that SPL13RNAi-6 plants had a significantly higher basal monomeric anthocyanin level under a well-watered condition (Fig. 5c). Interestingly, all SPL13RNAi genotypes accumulated a higher level of total monomeric anthocyanin during drought stress while levels in EV did not change (Fig. 5c). A comparable total polyphenol content was mainatined by all genotypes regardless of whether the plants were under well-watered or drought conditions (Fig. 5d).

\section{Flavonoid- and photosynthesis-related genes are enhanced in SPL13-silenced plants}

To understand whether the observed increase in the level of total monomeric anthocyanin and maintenance of photosynthesis efficiency under drought stress is regulated at the transcript level we analysed the expression 


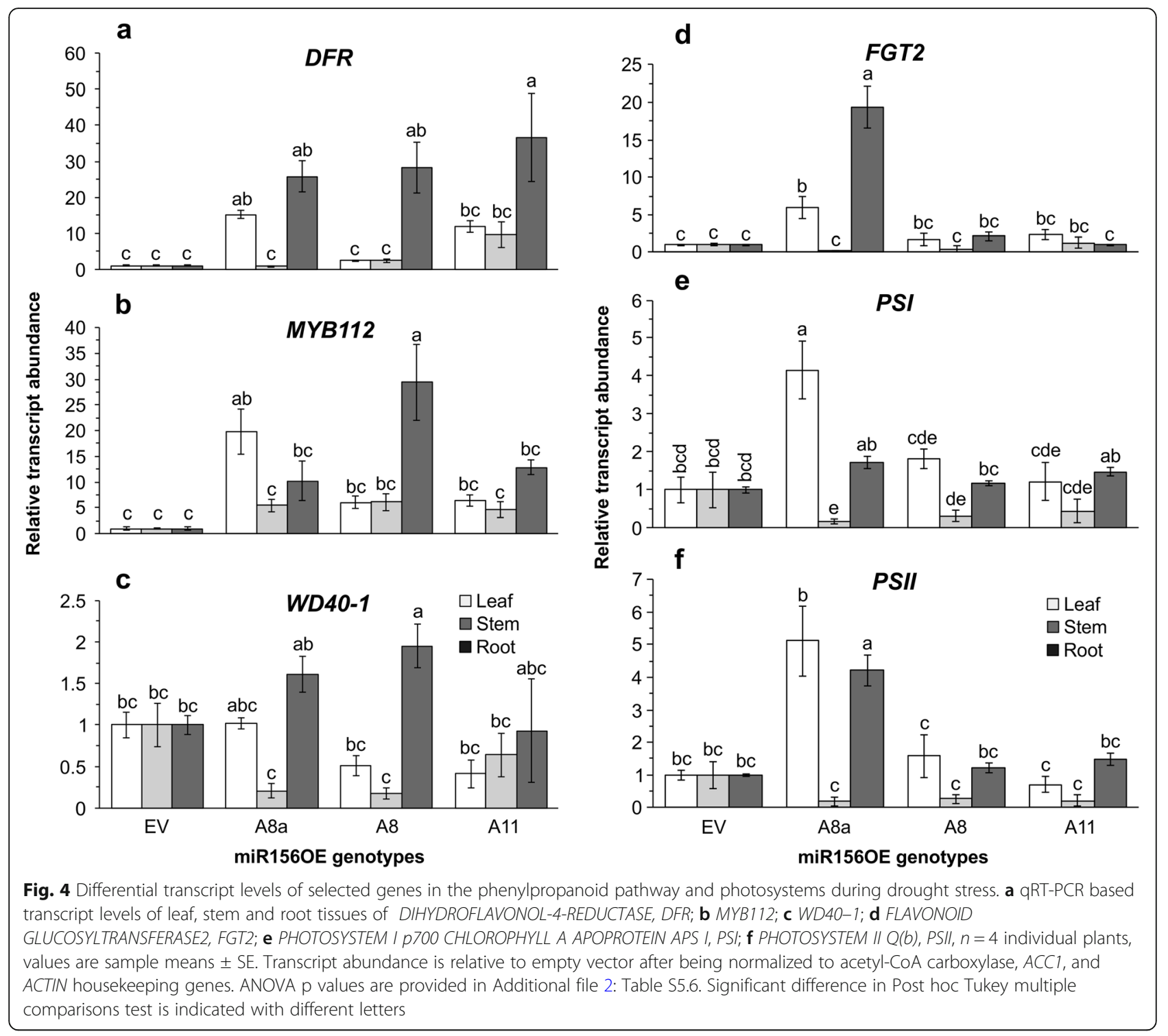

levels of anthocyanin-related and dehydration responsive genes. Our results showed that there were significant differences between genotypes under drought and control conditions (Fig. 5e-h and Additional file 2: Table S5.7). As expected, the transcript level of PHENYLALANINE AMMONIA-LYASE, PAL, the first committed step in the phenylpropanoid pathway, was significantly higher in two out of three SPL13RNAi genotypes (Fig. 5e). Similarly, DFR and FGT2 were also higher in two out of three SPL13RNAi genotypes (Fig. 5e,f). These consistently higher levels of $P A L, D F R$ and FGT2 transcripts suggest that the induction of flavonoid biosynthesis in response to drought stress is regulated by SPL13. In addition, the DEHYDRATION RESPONSIVE $R D$-22-LIKE (DRR) gene, which is regulated by MYB and MYC transcription factors and induced by drought and ABA [50, 51], was also expressed four- to 17 -fold higher in SPL13RNAi plants (Fig. 5f). In line with that, the transcription factor WD40-1 was increased three- to 14-fold in SPL13RNAi plants during drought stress (Fig. 5g). For photosynthesis-related genes, we analysed the transcript levels of PSI and PSII and found a two- to 10-fold and six to 43-fold increase in expression levels, respectively, in SPL13RNAi plants relative to EV (Fig. 5h), consistent with results in A8a and A8 genotypes (Fig. 4e, f).

\section{SPL13 is a direct regulator of DFR}

miR156 regulates the expression level of SPLs including SPL13 in alfalfa [8]. Given that DFR has four putative SBD binding motifs with core GTAC sequence in the promoter region (Fig. $5 \mathrm{i}$ and Additional file 2: Figure S3), we studied the occupancy of SPL13 in the promoter region of DFR using ChIP-qPCR in p35S:SPL13-GFP plants. The transgenic (p35S:SPL13-GFP) alfalfa plants 

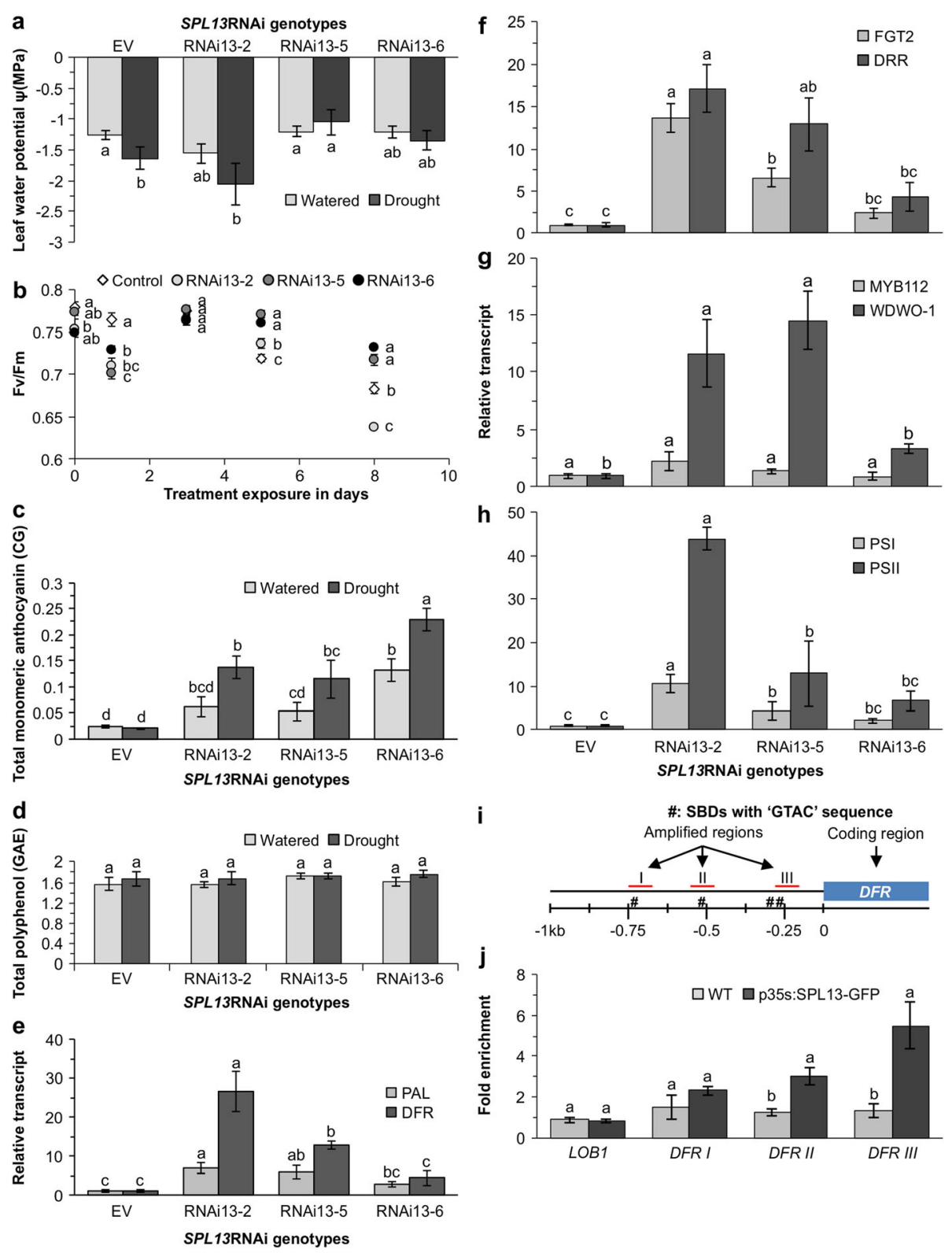

Fig. 5 SPL13 silencing regulates drought by coordinated metabolite, transcript, and physiological adjustments. a Leaf water potential in SPL13RNAi and EV plants; $\mathbf{b}$ dark adapted chlorophyll florescence, Fv/Fm, during drought stress; $\mathbf{c}$ total monomeric anthocyanin expressed as cyanidin-o-glucoside equivalent (CG); and $\mathbf{d}$ total polyphenol content expressed as gallic acid equivalent (GAE); e transcript levels of PHENYLALANINE AMMONIA-LYASE, PAL, and DIHYDROFLAVONOL-4-REDUCTASE, DFR; $\mathbf{f}$ FLAVONOID GLUCOSYLTRANSFERASE2, FGT2, and DEHYDRATION RESPONSIVE RD-22-LIKE, DRR; $\mathbf{g}$ MYB112 and WD40-1 transcription factor genes from the phenylpropanoid pathway in stems of SPL13RNAi and EV genotypes; $\mathbf{h}$ transcript levels of PHOTOSYSTEM I p700 CHLOROPHYLL A APOPROTEIN APS I, PSI, and PHOTOSYSTEM II Q(b), PSII under drought stresS; i schematic representation of potential SPL13 binding sites in the promoter region of DFR, $\mathbf{j}$ ChIP-qPCR based fold enrichment analysis of SPL13 in p35S:SPL13-GFP and WT plants from means of $\mathrm{n}=$ three individual plants where LATERAL ORGAN BOUNDARES-1, LOB1, is used as a negative control. Values are means $\pm \mathrm{SE}$, light gray bars in ' $\mathbf{a}$ ', ' $\mathbf{c}$ ' and ' $\mathbf{d}$ ' represent values under well-watered condition while dark gray bars represent values under drought stressed conditions. Relative transcript levels in ' $\mathbf{e}^{\prime}, \mathbf{\prime} \mathbf{\prime}$ ', ' $\mathbf{g}$ ' and ' $\mathbf{h}$ ' are shown relative to EV after being normalized to acetylCoA carboxylase, ACC1, and ACTIN housekeeping genes. ANOVA p values are provided in Additional file 2: Table S5.2, S5.7 and S5.8. Significant difference in Post hoc Tukey multiple comparisons test is indicated with different letters. Letters in multiple time point data of 'b' is analyzed separately

were developed previously by our group [52]. We selected three regions (I, II \& III) with the conserved SBD core sequences located at 750, 544 and $260 \mathrm{bp}$, respectively, upstream of the translation start codon of $D F R$ as potential SPL13 binding sites, and we tested them for SPL13 occupancy. LATERAL ORGAN BOUNDARIES$L I K E 1, L O B 1$, was used as a negative control for ChIPqPCR due to the low SPL13 binding ability to this gene 
despite the presence of a putative SBD sequence [52]. Compared to WT, p35S:SPL13-GFP plants were significantly higher in SPL13 binding to the DFR promoter region (Fig. 5j and Additional file 2: Table S5.8). There is a preferential binding of SPL13 towards the two most downstream putative SBD regions (II \& III) in the DFR promoter while region I did not show strong binding (Fig. 5i, j and Additional file 2: Figure S3). Of the three regions, region III showed the strongest binding to SPL13 (Fig. 5i, j), indicating that SPL13 could bind directly to $D F R$ to regulate its expression.

\section{WD40-1 positively regulates DFR expression and drought tolerance}

With the observed higher expression level of WD40-1 and flavonoid accumulation in miR156OE genotypes during drought stress and a finding from literature regarding the involvment of WD40-1 in the phenylpropanoid pathway [53], we aimed to investigate whether miR156 or SPL13 directly regulate the expression of WD40-1. Hence, we investigated the presence of conserved SPL binding (SBD) motifs in the promoter region of WD40-1. We used genome walking (GenomeWalker Clonetech Laboratories, Inc.) to obtain the promoter region sequence of WD40-1. However, we could not find either a miR156 target sequence or a SBD motif and thus concluded an indirect regulation of WD40-1 by miR156 or SPL13 (Additional file 2: Figure S4).

To further understand the potential role of WD40-1 in alfalfa drought tolerance, we generated plants with overexpressed (OE) or silenced (RNAi) WD40-1 and exposed these plants to drought stress. We used four different event-derived plants of WD40-1OE (OE04, OE09, OE14 and OE15) and WD40-1RNAi (RNAi03, RNAi04, RNAi10 and RNAi11) in comparison to WT plants (Fig. 6a, b). WD40-1 overexpressing genotypes were drought tolerant while the RNAi silenced WD40-1 genotypes were susceptible to drought stress (Fig. 6a, Additional file 2: Table S5.3). We investigated phenotypic and physiological responses such as root development, cholorophyll concentration and leaf water potential during drought stress and well-watered conditions.

WD40-1OE genotypes maintained a higher leaf water potential during drought stress (Fig. 6c) as compared to WT and WD40-1RNAi genotypes (data not shown). WD40-1OE genotypes developed longer roots and associated root weight (Fig. 6d, e, Additional file 2: Table S5.3). Moreover, WD40-1OE genotypes also maintained higher level of leaf chlorophyll concentration during drought stress (Fig. 6f, Additional file 2: Table S5.3).

To understand the role of WD40-1 in regulating drought stress through possible interaction with DFR and other genes in the phenylpropanoid/flavonoid pathway [24], we measured transcript levels of phenylpropanoid-assosciated genes under drought and well-watered conditions in WD40-1 silenced and overexpressing genotypes. Accordingly, an increase in WD40-1 expression enhanced DFR, PAL and FGT2 transcripts during drought stress while levels similar to that of WT were observed when plants were kept under well-watered condition (Fig. 7a, b, c, Additional file 2: Table S5.8). Moreover, the ABA-related dehydration responsive gene, $D R R$, and photosynthesis related genes, PSI and PSII, were increased in WD40-1OE genotypes compared to WD40-1RNAi and WT plants (Fig. 7d, e, f, Additional file 2: Table S5.8).

\section{Discussion}

Drought is one of the main factors that impair plant growth and development [54]. Plants respond to drought by showing deleterious effects, or by engaging in adaptive responses involving various molecular, biochemical and physiological strategies [55-57]. In this study, we used miR156OE, WD40-1OE, WD40-1RNAi, SPL13RNAi and GFP-tagged SPL13 genotypes to investigate the molecular and physiological strategies used by miR156 to regulate drought stress in alfalfa.

\section{Moderate levels of miR156 overexpression, WD40-1 overexpression or SPL13 silencing are sufficient to induce phenotypic and physiological drought tolerance strategies in alfalfa}

Of the different plant organs that respond to soil water deficit, roots are first to encounter changes in the rhizosphere. Findings in model plants showed initiation and elongation of lateral roots in drought tolerant genotypes to improve water uptake $[58,59]$. In this study, we observed a significant increase in root length accompanied by higher root biomass in plants moderately overexpressing miR156 (A8a and A8) and WD40-1. This is associated with a reportedly enhanced level of ABA [21] in miR156 overexpressing genotypes under drought stress. ABA enhances primary and lateral root development by regulating the expression of LATERAL ROOT ORGAN DEFECTIVE (LATD) gene [60]. Moreover, miR156 contributes to root development by silencing AtSPL10 to decrease the expression of AGAMOUS-LIKE MADS box protein 79 (AGL79) in Arabidopsis [61]. Accordingly, the enhanced root development under drought stress helps alfalfa plants to better access water from deeper soil surface. This finding is consistent with our previous report that showed increased root length in miR156OE and SPL13RNAi genotypes under drought conditions [21]. Moreover, moderate miR156OE, SPL13RNAi and WD40-1OE genotypes had higher leaf water potential despite their exposure to drought conditions. The observed drought tolerance in miR156OE (A8a and A8), WD40-1OE and SPL13RNAi genotypes 


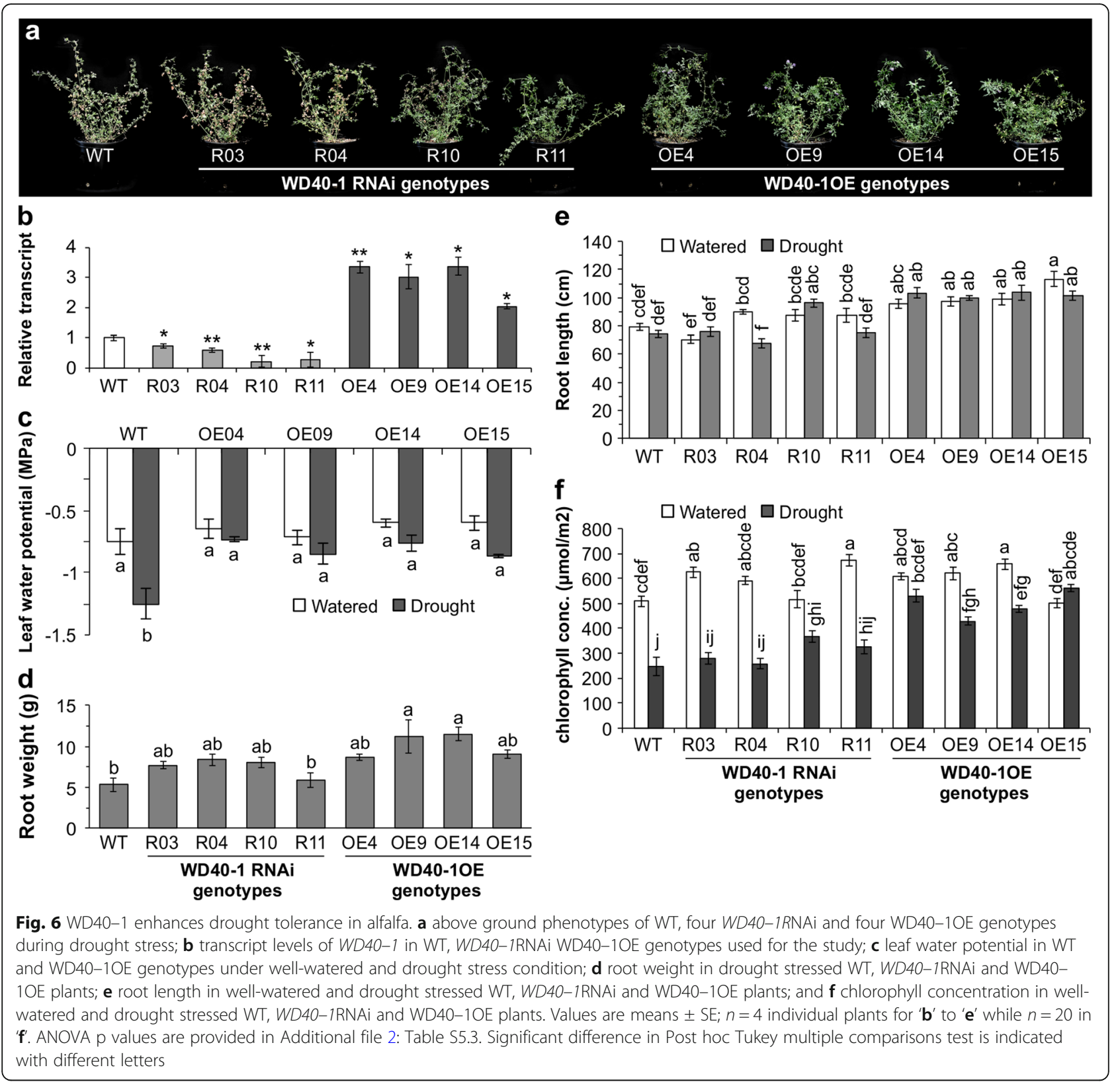

suggests this trait is at least partially negatively regulated by SPL13 and positively by miR156 and WD40-1.

Photosynthesis is negatively impacted by drought stress in alfalfa and other plant species [34, 62]. Of the many photosynthesis efficiency parameters, $\mathrm{Fv} / \mathrm{Fm}$ reflects the maximum quantum efficiency of PSII photochemistry possible in a dark-adapted state, and is considered a good stress indicator in plants [63-67]. Therefore, maintaining a higher Fv/ Fm was one of the parameters used in selecting abiotic stress tolerant cultivars of tomato and wheat $[64,68,69]$. The observed higher level of $\mathrm{Fv} / \mathrm{Fm}$ in $\mathrm{A} 8 \mathrm{a}$ and $\mathrm{A} 8$ genotypes in the current study suggests that their leaves may have a functional photosynthetic unit, in agreement with the observed maintained photosynthesis assimilation rate under drought. The observed higher Vcmax (Rubisco carboxylase activity) and $\operatorname{Imax}$ (electron transport rate) in A8a and A8 under drought further illustrate the maintenance of their photosystem despite drought stress. Such physiological adjustments were low to absent in A11 plants which showed susceptibility to drought stress. We also observed a higher Fv/Fm ratio in SPL13RNAi-5 and SPL13RNAi-6, which is consistent with our previously reported finding of increased photosynthetic assimilation rate in droughttreated SPL13RNAi genotypes [21]. This suggests that the maintenance of a higher photosynthetic assimilation rate, Vcmax, Jmax and high $\mathrm{Fv} / \mathrm{Fm}$ ratio 


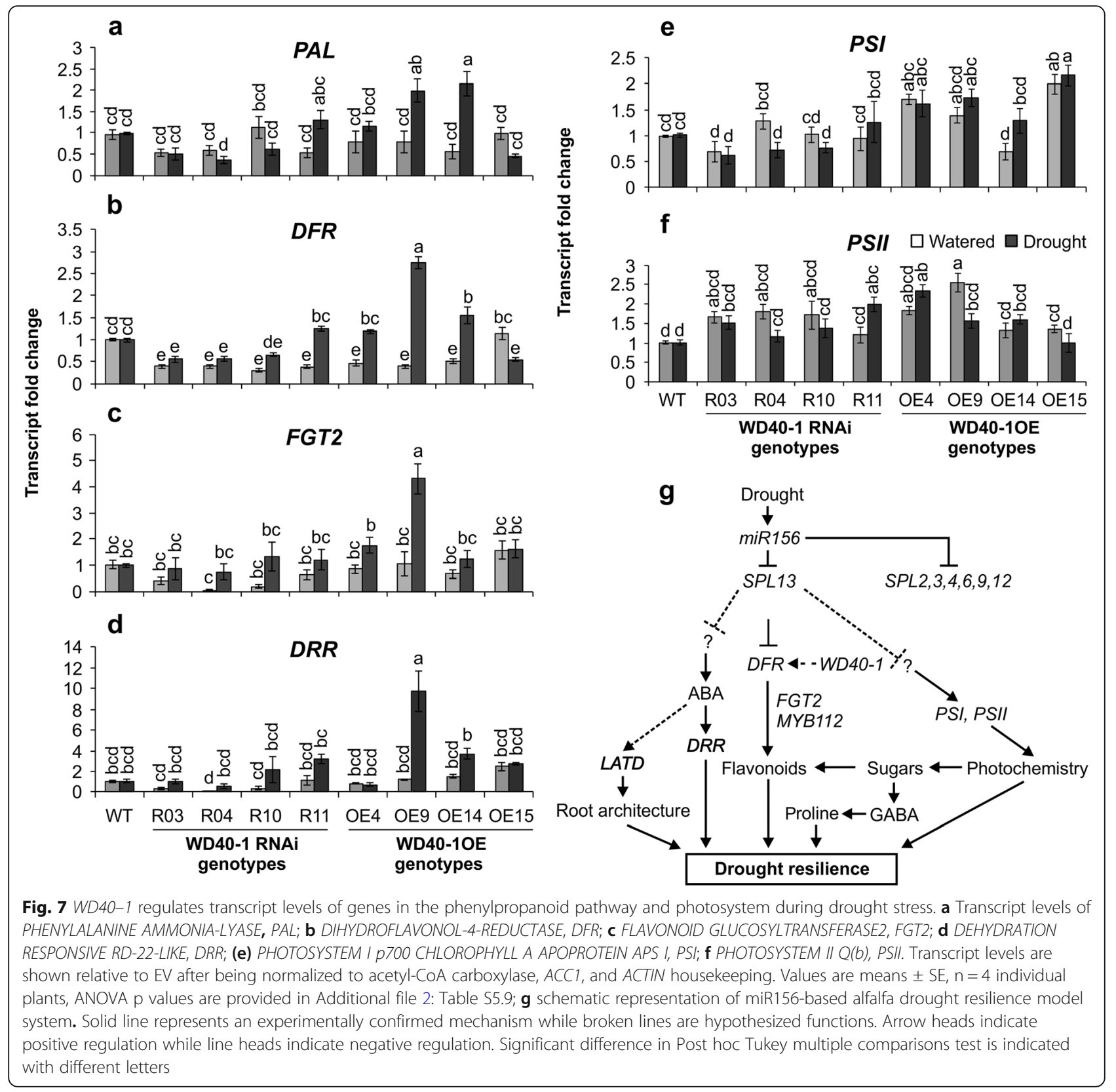

during drought stress in miR156OE and WD40-1OE genotypes may be regulated at least in part by SPL13 and WD40-1.

\section{miR156 overexpression enhances accumulation of stress- related metabolites}

The impact of environmental perturbations on plant metabolism varies among plant species, cultivars, and tissues considered [70]. Accumulation of specific secondary and transient primary metabolites (primary metabolites that are direct precursors of secondary metabolites) in various tissues are used in part to mitigate drought stress [27, 28, 71, 72]. Naya et al. [73] indicated the role of carbon metabolism and oxidative damage on nitrogenase activity reduction during moderate and higher drought stress levels in alfalfa. Other studies in M. truncatula, have shown a decrease in symbiotic nitrogen fixation under drought stress resulting in low levels of nitrogen-based metabolites [74].

In our study, alfalfa with a moderately enhanced expression of miR156 caused accumulation of anthocyanins, flavonols, and proteinogenic amino acids in leaf and stem tissues. The accumulation of these metabolites may help the plant to scavenge ROS produced during drought stress [40]. Moreover, these metabolites could help the plants to reduce water loss, and further absorb 
any remaining tightly bound water from the soil by lowering the osmotic balance in the root tissues. The high level of GABA in leaf, stem and root tissues of A8a and A8 should maintain a carbon-to-nitrogen balance through a GABA shunt bypassing the decarboxylation part of the TCA cycle [29]. The importance of GABA in mediating abiotic stress has been well documented in various plant species, including Arabidopsis [75], black pepper [76] and bentgrass [77]. Proline was also increased in A8a and A8 but not in A11 roots to regulate osmotic homeostasis as reported in other studies [21, 26]. The relatively lower level of proline abundance in roots of the highest miR156 overexpressor, A11, might have prevented these plants from maintaining high water levels in their system (Fig. 1g). The higher level of fructose and arabinose in leaf and stem tissues of droughttreated moderate miR156 expressors respectively could provide an energy source and/or an osmolyte. The higher sugar level suggests an actively functioning photosynthetic assimilation with the potential to supplement a carbon source for downstream metabolites. This is consistent with a previous finding that drought-stressed alfalfa plants accumulate sugars [78]. Moreover, the increased total monomeric anthocyanin and comparable total polyphenol levels in SPL13RNAi genotypes illustrated a targeted enhancement of flavonoids at least partially governed by silencing SPL13 in alfalfa to scavenge ROS during drought stress.

\section{miR156, WD40-1 and SPL13 regulate phenylpropanoid} and photosystem genes under drought

Due to the various roles that polyphenols play in stress response, efforts have been made to increase their levels in many plants, including alfalfa [79]. Enhanced accumulation of flavonoids and proanthocyanidins (PA) in alfalfa has important quality implications for animal feed, as moderate amounts of PA tend to reduce bloating in ruminant animals [80-82]. In our study, we found that phenylpropanoid pathway-related genes are enhanced in moderately overexpressing miR156 alfalfa plants, which is consistent with the increase in anthocyanin and flavonol levels in these plants. DFR, WD40-1 and MYB112 were higher in A8a and A8 during drought, contributing to anthocyanin accumulation. Similarly, SPL13RNAi genotypes showed enhanced levels of DFR, FGT2 and $P A L$ transcripts associated with enhanced level of total monomeric anthocyanin, indicating enhancement of the phenylpropanoid/flavonoid pathway. In another study, Arabidopsis plants overexpressing miR156 accumulated anthocyanin in response to salt and mannitol (mimicking drought) treatments by increasing $D F R$ expression [23]. The enhanced DFR expression level in Arabidopsis was regulated by silencing SPL9 [23]. Our findings suggest that accumulation of anthocyanins and other polyphenols may be regulated via SPL13 in alfalfa. Moreover, the enhanced level of DFR in WD40-1OE plants and reduced in WD40-1RNAi plants suggests that DFR is positively regulated by the WD40-1 to promote flavonoid biosynthesis, but the mechanism of this regulation remains to be investigated.

To investigate whether the higher photosynthetic assimilation rate during drought stress in SPL13RNAi [21] and also WD40-1OE, WD40-1RNAi and miR156OE genotypes (current study) are regulated at the transcriptional level, we investigated expression of genes mediating photosynthesis. We found that PSI and PSII were significantly increased in moderately overexpressing miR156OE genotypes and SPL13RNAi genotypes upon drought. Previously, we reported an increased abundance of $\mathrm{ABA}$, which regulates stomatal aperture by active hydrolysis during drought stress in miR156OE A8 plants [21]. In the current study, we examined expression of the ABAinduced dehydration responsive gene (RD22) and found it to be significantly increased in SPL13RNAi plants during drought stress. The consistent observation of higher polyphenols and photosystem assimilation rate with associated transcripts during drought stress in moderate miR156OE and SPL13RNAi genotypes suggests a drought regulation strategy of miR156.

\section{SPL13 binds to DFR to regulate its expression and flavonoid biosynthesis}

To investigate whether the increased flavonoid accumulation and expression of phenylpropanoid-associated genes, especially $D F R$, are directly regulated by the miR156-SPL13 module, we conducted a ChIP-qPCR analysis to determine binding of SPL13 to DFR. DFR catalyses flavonoid biosynthesis by reducing dihydroflavonols to leucoanthocyanidins playing a critical role in anthocyanin biosynthesis [83]. A previous report showed SPL9 directly regulates the expression level of $D F R$ to enhance accumulation of anthocyanin in response to $\mathrm{NaCl}$ and mannitol treatment in Arabidopsis [23]. In the current study, we showed increased DFR expression during drought stress in moderately overexpressing miR156 and SPL13RNAi plants. Accordingly, we selected DFR to test for SPL13 binding, given the presence of multiple potential SBD core GTAC sequences in the DFR promoter. The fold enrichment from ChIP-qPCR showed the strongest SPL13 binding was observed at region III of the DFR promoter, which is located closest (260 bp) to the DFR coding sequence. This is in line with reports that showed the conserved core SBD element is not by itself sufficient for SPL binding, but also determined by the position of the SBD and the flanking DNA sequences [11, 84, 85]. SPL13 acts as a transcriptional suppressor of $D F R$ during drought stress as confirmed by higher expression of DFR in SPL13RNAi and miR156OE plants. 


\section{Conclusions}

We recently reported that miR156 regulates drought tolerance in alfalfa by silencing SPL13 [21]. Understanding the mechanisms deployed by miR156 in drought tolerance could be exploited as a tool in crops for marker-assisted breeding. In the current study, we investigated metabolomic, physiological and molecular mechanisms to show how low- to moderate levels of miR156 expression is sufficient to induce drought tolerance in alfalfa. Moderate levels of miR156 in genotypes of A8a and $A 8$ induced accumulation of stress mitigating metabolites, such as anthocyanins, flavonols, GABA, proline and others in the leaf, stem and root tissues. These metabolites could help the plants to scavenge ROS, reduce water loss and further absorb any remaining tightly bound water from the soil by lowering the osmotic balance in the root tissues. In addition, the plants showed physiological adjustments such as improved photosynthetic assimilation rate, maintained $\mathrm{Fv} / \mathrm{Fm}$ ratio, and enhanced root growth and development. The relatively low levels of stress mitigating metabolites and reduced physiological adjustments may have resulted in drought susceptibility in the highest miR156 overexpressor (A11). We also determined direct binding of SPL13 to the DFR promoter. SPL13 acts as a transcriptional suppressor of $D F R$ during drought stress as confirmed by higher expression of DFR in SPL13RNAi and miR156OE plants. Similar observation of SPLs suppressing the expression of DFR has been reported in Arabidopsis [86] where SPL9 silenced DFR in response to salt and mannitol treatment [23]. Moreover, we detected an increase in expression of genes involved in the phenylpropanoid and photosynthetic pathways, including $D F R$, MYB112, PSI and PSII in miR156OE plants under drought. DFR, FGT2, PSI and PSII were also increased in SPL13RNAi plants under drought stress.

We propose a model for a drought tolerance mechanism regulated by moderate levels of miR156 overexpression (Fig. 7g). The diagrammatic representation shows the central role of miR156 in regulating drought stress in alfalfa. MiR156 is induced by drought stress, which in turn silences SPL13 [21]. Reduced expression of SPL13 driven by miR156 and increased levels of WD40-1 enhance DFR resulting in accumulation of anthocyanins. In moderate miR156OE plants, primary metabolites such as GABA, proline and sugars also accumulate for carbon-to-nitrogen balance and osmotic homeostasis. Induction of miR156 during drought stress also enhances phenotypic plasticity, such as longer roots and higher biomass to access more water from the rhizosphere. With reduced SPL13 expression, miR156OE and WD40-1OE, higher photosynthesis efficiency is also achieved during drought stress. We conclude that moderate levels of miR156 expression silence SPL13 and increase WD40-1 expression to fine-tune DFR expression for anthocyanin biosynthesis and regulate various developmental, physiological and biochemical processes in alfalfa leading to improved drought resilience.

\section{Methods \\ Genetic material \\ miR156 overexpressing and SPL13RNAi plants}

Medicago sativa L. N4.4.2 plants [87] were obtained from Dr. Daniel Brown (Agriculture and Agri-Food Canada) and used as wild-type genotypes. Plants overexpressing miR156 (miR156OE) at different levels (A8a, $\mathrm{A} 8$ and A11) and an empty vector control (EV) were generated previously in our laboratory and used in this experiment [13]. miR156 is slightly (0.5) elevated in A8a, but it is moderate (1.5) to higher (2.5) relative transcript level in A8 and A11, respectively [13]. The plants were grown in a fully automated greenhouse with 16-h light (380-450 $\left.\mathrm{W} / \mathrm{m}^{2}\right)$, relative humidity $(\mathrm{RH})$ of $70 \%$ and temperature of $25 \pm 2{ }^{\circ} \mathrm{C}$ at the Agriculture and Agri-Food Canada London Research and Development Center, London, Ontario, Canada. Given that alfalfa is an obligatory outcross, we used vegetative cuttings for propagation according to Aung et al [13] to maintain genotypes throughout the study. Since miR156 down-regulates seven $S P L$ genes (including SPL13) to regulate a network of downstream genes, we used SPL13RNAi genotypes (SPL13RNAi-2, SPL13RNAi-5 and SPL13RNAi-6) [21] selected for their low SPL13 expression levels relative to wild-type alfalfa and other SPL13RNAi transgenic alfalfa plants.

\section{Generation of WD40-1 overexpressing and WD40-1RNAi alfalfa plants}

Four WD40-1OE (OE04, OE09, OE14 and OE15) and four WD40-1RNAi (R03, R04, R10 and R11) genotypes were generated to investigate the role of WD40-1 in drought tolerance. WD40-1 overexpression and downregulated genotypes were generated using constructs made from alfalfa homolog WD40-1 (Medtr3g074070) using Gateway cloning system (Thermo Fisher Scientific, Mississauga $\mathrm{ON}$ ). For overexpression studies, full-length WD40-1 was amplified from alfalfa (Medicago sativa) cDNA using primers with AttB sites attached, forward (B1-WD40-1) and reverse (B2-WD40-1) (Additional file 1: Table S1) and cloned into the pDONR/Zeo entry vector. For downregulation studies, a $253 \mathrm{bp}$ putative WD40-1 fragment was amplified from alfalfa cDNA using AttB sites attached forward (B1-WD40-1-RNAi) and reverse (B2-WD40-1-RNAi) (Additional file 1: Table S1) primers and cloned into $\mathrm{pDONR} / Z$ eo entry vector.

After PCR screening and confirmation by sequencing, LR reactions were performed for the overexpression and RNAi constructs to recombine the fragments into the pMDC83 (overexpression) and pHELLSGATE12 
(RNAi) vectors. Subsequently, overexpression and RNAi constructs were used to transform Agrobacterium tumefaciens strain EHA105 which was then used to transform alfalfa. QRT-PCR was then used to analyze WD40-1 gene expression in WD40-1-OE WD40-1-RNAi genotypes using primers WD1-qPCR-F and WD1-qPCR-R (Additional file 1: Table S1).

\section{Imposing drought stress}

Drought stress was imposed on alfalfa plants devoid of water for 2 weeks at 30 days post vegetative propagation (juvenile vegetative stage) during which time plants were kept in a completely randomized design. Equal soil moisture levels were maintained before starting the experiment using a SM 100 soil moisture sensor (Spectrum Technologies Inc., Jakarta, Indonesia). At least four biological replicates were used per genotype per treatment for transcript and metabolite analysis, while 4 to 10 plants were used for physiological analysis (each replicate being an individual plant). The entire experiment was repeated under the same growth and drought stress conditions to test the repeatability of results. Leaves (newly developed upper leaves), stems (lower $5 \mathrm{~cm}$ internode close to soil) and roots $(7.5 \mathrm{~cm}$ of main and auxiliary root tips) were harvested from miR156OE, SPL13RNAi, WD40-1OE, WD40-1RNAi, EV and wild-type plants depending on the experiment. Samples were flash frozen with liquid nitrogen and kept at $-80^{\circ} \mathrm{C}$ for later metabolomic and transcriptomic analyses.

\section{Metabolite extraction for parallel LCMS and GCMS analysis}

To explore miR156-related regulation of secondary metabolites and transient primary metabolites, extracts of stem, leaf and root tissues of drought-stressed miR156OE and control plants were subjected to Liquid Chromatography-Mass Spectrometry (LCMS) and Gas Chromatography-Mass Spectrometry (GCMS) analysis. Extraction of samples was performed according to Ayenew et al. [28] for parallel LCMS and GCMS analysis. Unless stated otherwise, chemicals used for the analysis were obtained from Sigma-Aldrich, Canada. Briefly, frozen $50 \mathrm{mg}$ tissues were crushed with a RETCH-mill (Retsch Gmbh, 42,787 Haan, Germany) and stainlesssteel beads. One milliliter prechilled extraction solution, methanol/chloroform/water $(2.5 / 1 / 1 \mathrm{v} / \mathrm{v} / \mathrm{v})$, was added containing an internal standard Ribitol/adonitol 0.225 $\mathrm{mg} / \mathrm{mL}$ for GCMS analysis while ampicillin (Sigma, and Saint Luis, Missouri, USA) and corticosterone at $1 \mathrm{mg} /$ $\mathrm{mL}$ for LCMS to normalize extraction variability. The mixture was vortexed and ultra-sonicated for $10 \mathrm{~min}$. Following centrifugation at $14000 \mathrm{rpm}$ for $10 \mathrm{~min}$ (at $4^{0}$ C), supernatant was collected and mixed with equal volumes of $300 \mu \mathrm{L}$ water and chloroform. The mixtures were vortexed briefly and centrifuged at $14000 \mathrm{rpm}$ for 5 min to collect the upper aqueous phase for parallel LCMS and GCMS analyses.

LCMS analysis was performed using an Agilent 1290 Infinity LC system coupled with a Thermo Q-Exactive Quadrupole-Orbitrap mass spectrometer. Analytes were separated with an Agilent Eclipse Plus C18 ZORBAX Rapid Resolution High Definition (RRHD) $1.8 \mu \mathrm{m}$ particle 2.1 i.d. X $50 \mathrm{~mm}$ column. The instrument was equipped with electrospray ionization (ESI) interface operating in a negative and positive ion mode for better metabolite identification. Metabolites were identified based on mass to charge ratio $(\mathrm{m} / z)$, retention time and fragmentation pattern in comparison to commercial standards, ChemSpider and ReSpect phytochemical databases [28, 71]. MZmine2 software [88] was also used for LCMS metabolite mass detection, chromatogram building, and the separation of overlapping peaks. In parallel, transient primary metabolites were explored using $75 \mu \mathrm{L}$ aliquots of the extracted samples for LCMS using an Agilent 5975C Triple-Axis Detector MSD and 7890A GC system in splitless mode. The aliquots were dried using an Eppendorf Vacufuge $^{\text {Th }}$ concentrator (Hamburg, Germany), derivatized by $40 \mu \mathrm{L} \mathrm{O}$-methylhydroxylamine hydrochloride in pyridine with $7 \mu \mathrm{L}$ standard alkane mixture $(0.029 \% \mathrm{v} / \mathrm{v} \mathrm{C} 10$ $\mathrm{C} 20$ of each $50 \mathrm{mg} / \mathrm{l})$ for $2 \mathrm{~h}$ at $37^{\circ} \mathrm{C}$ followed by $70 \mu \mathrm{L} \mathrm{N}$ methyl- $\mathrm{N}$-[trimethylsilyl] trifluoroacetamide (MSTFA) for silylation. Metabolites from GCMS were identified using the retention time of the standard alkane mixture with their mass spectra and a NIST 2011 mass spectral library $[27,28,72]$.

\section{Total monomeric anthocyanin and polyphenol determination}

Total monomeric anthocyanin, TMA, and total polyphenol, TPP, were determined using a $\mathrm{pH}$ deferential extraction method [89, 90]. Briefly, flash-frozen in liquid nitrogen samples were crushed with mortar and pestle under liquid nitrogen and $500 \mathrm{mg}$ tissue were used for the combined analysis of TMA and TPP. Samples were treated with $2 \mathrm{ml}$ acidified methanol $(\mathrm{MeOH}$ with $1 \%$ $\mathrm{HCL}$ ), vortexed and sonicated at $20 \mathrm{KHz}$ for $15 \mathrm{~min}$. Homogenate was stirred at 3000 RPM for $1 \mathrm{~h}$ and centrifuged (at $4{ }^{\circ} \mathrm{C}$ ) for $10 \mathrm{~min}$ at full speed $(14,000$ RPM). The supernatant was collected, added $2 \mathrm{ml}$ chloroform, vortexed and centrifuged at full speed for $10 \mathrm{~min}$. The upper aqueous phase was collected, filtered with Whiteman 0.2 um filters, and divided into three equal aliquots for TMA (pH 1.0 and 4.5) and TPP analysis. The first aliquot was mixed with an equal volume of $0.025 \mathrm{M} \mathrm{KCl}$ at $\mathrm{pH} 1.0$ while the second is mixed with equal volumes of $0.4 \mathrm{M}$ sodium acetate at $\mathrm{pH} 4.5$ and measured absorbance at 520 and $700 \mathrm{~nm}$ with water as a blank. TPP was analysed by mixing an equal volume of 
the third aliquot with Folin-chiocalteu reagent (diluted 1:10 with water) and vortexed for $3 \mathrm{~min}$. Four $\mathrm{ml}$ of sodium carbonate $(7.5 \% \mathrm{w} / \mathrm{v})$ was added to the mixture, which was then vortexed and incubated for $30 \mathrm{~min}$ in the dark. TPP was determined as gallic acid equivalent (GAE) after measuring absorbance of the aliquot at 765 $\mathrm{nm}$ with acidified methanol as blank. TMA level is expressed as mg cyanidin-3-o-glucoside (CG) equivalent.

\section{Physiological and phenotypic data measurement}

To determine drought mitigating strategies, we investigated phenotypic and physiological parameters. Midday photosynthesis assimilation rates and dark-adapted chlorophyll fluorescence $(\mathrm{Fv} / \mathrm{Fm})$ were measured in newly growing upper unshaded leaves using a LI-6400XT portable photosynthesis meter coupled with Fluorescence System (LI-COR Biosciences, Lincoln, Nebraska, USA). Photosynthetic assimilation rate responses across a gradient of $\mathrm{CO}_{2}$ level $(\mathrm{A} / \mathrm{Ci})$ in the mesophyll cells to determine the maximum rate of rubisco carboxylase activity (Vcmax) and maximum photosynthetic electron transport rate $(\max )$ was calculated to determine photosynthetic efficiency using the $\mathrm{R}$ statistical software plantecophys package [91]. Chlorophyll concentration index (CCI) of newly growing upper leaves were also determined using an Apogee MC100 instrument (Apogee instruments, Logan, Utah, USA) [92]. To determine plant water status, the midday leaf water potential was measured using a SAPS II Portable Plant Water Status Console (Soilmoisture Equipment Corp., Santa Barbara, CA, USA) in darkadapted leaves by covering leaves with a polyethylene bag and aluminium foil for $20 \mathrm{~min}$. In addition, above and below ground phenotypic parameters were measured, such as stem number and shoot weight, root length and weight according to Aung et al. [13], and stem basal diameter at $1 \mathrm{~cm}$ above stem-soil interface.

\section{RNA extraction and qRT-PCR analysis}

Stem, leaf and root samples were collected and flash frozen in liquid nitrogen and kept in a $-80^{\circ} \mathrm{C}$ freezer until further use. Approximately $50 \mathrm{mg}$ fresh weight was used for total RNA extraction using a PowerPlant ${ }^{\circ}$ RNA isolation kit (Cat \# 13500) for leaf samples, a QIAGEN RNeasy ${ }^{\circ}$ Plant mini kit for stem and root tissues (Cat \# 74904), and a PowerLyzer 24 bench top bead-based homogenizer (Cat \# 13155) following manufacturers protocols. The extracted RNA was treated with Ambion TURBO DNA-free ${ }^{\mathrm{Tw}}$ DNase (Cat \# AM1907) followed by iScript $^{\text {tw }}$ cDNA synthesis (Cat \# 1708891).

Transcript levels of selected genes involved in secondary metabolite biosynthesis and photosynthesis were investigated in this study. Using publicly available transcriptomics data of two miR156OE alfalfa genotypes under control (unstressed) conditions [8] and M. truncatula genome sequence Mt4.0 V2 (http://www.medicagogenome.org/ downloads), transcripts of differentially expressed genes with the SBD core GTAC sequence within $2.5 \mathrm{~kb}$ of their promoter regions were identified. Among those, genes shown by Gene Ontology analysis to be involved in flavonoid biosynthesis, photosynthetic efficiency and stress tolerance were chosen for expression analysis by qRT-PCR. Primers specific to the above genes (Additional file 1: Table S1) were designed using $M$. truncatula genome sequence and amplified product was sequenced for an identity check (Additional file 2: Figure S2). Publicly available Primer3 software (http://primer3.ut.ee/) was used to design primers, and their efficiency was verified at different concentrations with gradient annealing temperature PCR before using for qRT-PCR analysis.

QRT-PCR was performed using the CFX96 ${ }^{\text {mi }}$ RealTime PCR detection system and SsoFast ${ }^{\mathrm{sx}}$ EvaGreen $^{\circ}$ Supermixes (Bio-Rad Cat \# 1725204). Specifically, $2 \mu \mathrm{L}$ cDNA (equivalent to $200 \mathrm{ng} \mathrm{cDNA}), 1 \mu \mathrm{L}$ forward and reverse gene-specific primers (10 $\mu \mathrm{M}$ each), $5 \mu \mathrm{L}$ SsoFast Eva green Supermix, and $2 \mu \mathrm{L}$ of nuclease-free water was used to make the final reaction volume of $10 \mu \mathrm{L}$. PCR amplification was performed at: cDNA denaturation at $95^{\circ} \mathrm{C}$ for $30 \mathrm{~s}$ followed by 40 cycles of $95^{\circ} \mathrm{C}$ for $10 \mathrm{~s}$, $58^{\circ} \mathrm{C}$ for $30 \mathrm{~s}$ and $72^{\circ} \mathrm{C}$ for $30 \mathrm{~s}$ (denaturation, annealing and extension, respectively) followed by a melting curve that runs from $65^{\circ} \mathrm{C}$ to $95^{\circ} \mathrm{C}$ with a gradual increment of 0.5 per $5 \mathrm{~s}$. All reactions were performed with three technical and four biological replicates. Transcript levels were analysed relative to acetyl-CoA carboxylase $(A C C 1)$ and ACTIN housekeeping genes designed based on alfalfa sequence $[13,21]$.

\section{ChIP-qPCR analysis of SPL13-DNA binding}

Shoot tips of alfalfa plants overexpressing SPL13 tagged with GFP driven by the CaMV35S promoter (p35S:SPL13GFP) [52] were used to understand the occupancy of SPL13 on promoters of downstream genes contributing to drought tolerance. One-month-old SPL13-GFP overexpressing genotypes and WT control plants were used for ChIP-qPCR analysis based on previously published protocol [93] with some modifications. Briefly, $500 \mathrm{mg}$ of shoot tips from WT and p35S:SPL13-GFP plants were collected, washed, proteins bound to DNA were cross-linked using $1 \%$ formaldehyde and mixtures were ground with liquid nitrogen. Extraction reagents and buffers are listed in Additional file 2: Table S4. Powdered tissues were homogenized with $15 \mathrm{ml}$ of prechilled Extraction Buffer 1 and filtered with two layers of Miracloth (Millipore, Canada). Subsequently, the filtered mixture was centrifuged at $3000 \mathrm{~g}$ for $20 \mathrm{~min}$ and supernatant was discarded while the pellets were resuspended in $1 \mathrm{ml}$ of prechilled Extraction Buffer 2 and centrifuged at $12000 \mathrm{~g}$ for $10 \mathrm{~min}$. Afterwards, pellets were resuspended in $300 \mu \mathrm{L}$ prechilled 
Extraction Buffer 3 and centrifuged at $16000 g$ for $1 \mathrm{~h}$. The supernatant was removed, and chromatin pellets were resuspended in $300 \mu \mathrm{L}$ of Nuclei Lysis Buffer by gentle pipetting and sheared twice at power 3 for $15 \mathrm{~s}$ on ice using a Sonic Dismembrator (Fisher Scientific, USA). Twenty microliter of supernatant aliquots were kept aside for later use as an input DNA control while using the remaining solution for immunoprecipitation. Chromatin solution was brought to $1.5 \mathrm{~mL}$ using a ChIP dilution buffer and divided into two equal parts for chromatin immunoprecipitation and a negative control. To each tube, $30 \mu \mathrm{L}$ of protein $\mathrm{A}$-agarose beads (Millipore, Canada) were added and the mixture was gently agitated, centrifuged $(3500 \mathrm{~g})$ for $1 \mathrm{~min}$, and supernatant was transferred for immunoprecipitation while discarding the beads. Five $\mu \mathrm{L}$ of Ab290 GFP antibody was added to one of the chromatin solutions (keeping the second one as a no-antibody negative control) for an overnight gentle agitation at $4{ }^{\circ} \mathrm{C}$. After $12 \mathrm{~h}, 40 \mu \mathrm{L}$ of protein A-agarose beads were added and immune complexes were recovered by centrifugation and washed with cycle of low normality salt, high salt, $\mathrm{LiCl}$ and TE buffer. Immunocomplexes were eluted from beads using $250 \mu \mathrm{L}$ of Elution Buffer and cross linking was reversed with $20 \mu \mathrm{L}$ of $5 \mathrm{M} \mathrm{NaCl}$ incubated at $65^{\circ} \mathrm{C}$ for 5 h. To each sample $10 \mu \mathrm{L} 0.5 \mathrm{M}$ EDTA, $20 \mu \mathrm{L} 1 \mathrm{M}$ Tris$\mathrm{HCl}(\mathrm{pH} 6.5)$ and $2 \mu \mathrm{L}$ of $10 \mathrm{mg} / \mathrm{mL}$ proteinase K (SigmaAldrich, Canada) were added. DNA was extracted using phenol: chloroform $(1: 1, \mathrm{v}: \mathrm{v})$, recovered by precipitation with ethanol and $0.3 \mathrm{M}$ sodium acetate $(\mathrm{pH}=5.2)$ and $2 \mu \mathrm{L}$ glycogen carrier $10 \mathrm{mg} / \mathrm{mL}$ (Sigma-Aldrich, Canada) after overnight incubation at $-20^{\circ} \mathrm{C}$. After $12 \mathrm{~h}$, the solution was centrifuged at full speed for $20 \mathrm{~min}$ to pellet the DNA and pellet was then washed with $70 \%$ ethanol, resuspended with $16 \mu \mathrm{L}$ of distilled water, and DNA was used for ChIP-qPCR analysis. To obtain the DFR promoter region sequence from $M$. sativa, proDFR1-MTR primers (Additional file 1: Table S1) were designed using a close relative $M$. truncatula sequence and amplified region was cloned into TOP10 competent $E$. coli cells using CloneJET (Thermo Scientific) and sequenced. Subsequently, proDFR ChIP-qPCR primers (Additional file 1: Table S1) were designed based on alfalfa sequences. QRT-PCR was performed using ChIP-precipitated DNA as described above while fold enrichment was calculated by dividing $\mathrm{Ct}$ values of p35S:SPL13-GFP to WT and comparing with LOB1 reference gene [52].

\section{Genome walking for WD40-1 promoter nucleotide sequence}

Due to lack of alfalfa genome sequence, we used Clonetech GenomeWalker $^{\text {rs }}$ (California, USA Cat No. 638904) to obtain nucleotide sequence of the WD40-1 promoter region. In brief, we extracted genomic DNA from wild-type alfalfa plants using a Nucleospin ${ }^{\odot}$ Tissue DNA extraction kit
(MACHEREY-NAGEL Gmbh \& Co. KG Germany, Cat. No. 740952). GenomeWalker "libraries" were prepared by digesting the DNA with four different restriction enzymes (DraI, EcoRV, PvuII and StuI) at $37^{\circ} \mathrm{C}$ for 2 $\mathrm{h}$ to generate blunt ends. Subsequently, two nested PCR amplifications were performed sequentially for each library using gene specific primers (GSP1 and GSP2) and adapter primers (AP1 and AP2) from the kit (Additional file 1: Table S1). PCR products were analyzed on a $1.5 \%$ agarose gel followed by cloning into a pJET1.2 cloning vector to facilitate sequencing. Subsequently, sequences obtained from the four libraries were aligned together to generate the consensus promoter region sequence of WD40-1 in alfalfa.

\section{Statistical data analysis}

Shapiro-Wilk test were used for checking the normal distribution of data before proceeding to analysis of variance (ANOVA). Subsequently, Tukey post hoc multiple comparison were done on molecular (qRT-PCR and ChIP-qPCR), metabolomics (LCMS and GCMS), physiological and phenotypic data. Pair-wise t-test comparison was implemented between WD40-1OE and wild-type plants and with WD40-1RNAi plants for WD40-1 transcript abundance. Metabolite profile data were subjected to pareto scaling before principal component analysis (PCA) in which metabolites were mean-centred followed by dividing with the square root of the standard deviation. All statistical data analyses were undertaken using R-software environment 3.2.5.

\section{Supplementary information}

Supplementary information accompanies this paper at https://doi.org/10. 1186/s12870-019-2059-5.

Additional file 1: Table S1. List of primers used and their nucleotide sequences.

Additional file 2: Table S2. LCMS-based metabolite profiles of drought stressed alfalfa plants. Table S3 GCMS-based relative metabolite abundance in drought stressed alfalfa plants. Table S4 Buffers used in ChIP assay and their components. Table S5.1 Analysis of variance, ANOVA, $P$ values of data for phenotype and physiological responses in miR156OE genotypes and EV plants. Table S5.2 Analysis of variance, ANOVA, P values of data for phenotype, physiological and metabolite responses in SPL13RNAi genotypes and EV plants. Table S5.3 Analysis of variance, ANOVA, P values of data for phenotype and physiological responses in WD40-1OE, WD40-1RNAi and wild type plants. Table S5.4 Analysis of variance, ANOVA, $P$ values of data for LCMS-based metabolite profiling in miR1560E genotypes and EV alfalfa plants. Table S5.5 Analysis of variance, ANOVA, $P$ values $(P>F)$ of data for GCMS-based metabolite profiling in miR156OE genotypes and EV alfalfa plants. Table S5.6 Analysis of variance, ANOVA, $P$ values of data for qRT-PCR based transcript level in miR156OE genotypes and EV alfalfa plants. Table S5.7 Analysis of variance, ANOVA, P values of data for qRT-PCR based transcript level in SPL13RNAi genotypes and EV alfalfa plants. Table S5.8 Analysis of variance, ANOVA, $P$ values of data for ChIP-qPCR based transcript level in p35S:SPL13-GFP genotypes and Wild-type alfalfa plants. Table S5.9 Analysis of variance, ANOVA, P values of data for qRT-PCR based transcript level in WD40-1RNAi silenced and WD40-1over expressing plants. Figure 
S1 Stem colour development in miR1560E plants during drought stress. Figure S2 Alignment of sequences of amplified by q-PCR from Medicago sativa with those of their counterparts in Medicago truncatula. Figure S3 Promoter sequence of the alfalfa DIHYDROFLAVONOL-4-REDUCTASE (DFR) gene with putative SBD binding elements. Figure S4 Nucleotide sequence of the alfalfa WD40-1 promoter region.

\section{Abbreviations}

ABA: Abscisic acid; DAG: Delphinidin 3-O-(6"-acetyl)-glucoside; DFR: DIHYDROFLAVONOL-4-REDUCTASE; DRR: DEHYDRATION RESPONSIVE RD22-LIKE; EV: Empty vector; FGT2: FLAVONOID GLUCOSYLTRANSFERASE2; GABA: Gamma-aminobutyric-acid; GCMS: Gas chrmotaography mass spectrometry; LCMS: Liquid chromatography mass spectrometry; LOB1: LATERAL ORGAN BOUNDARIES-LIKE1; miR156: microRNA156: PA: Proanthocyanidins; PAL: PHENYLALANINE AMMONIA-LYASE; PAP1: PRODUCTION OF ANTHOCYANIN PIGMENT 1; PCA: Principal Component Analysis; PG: Peonidin 3-O-glucoside; PPP: Pentose phosphate pathway; PSI: PHOTOSYSTEM I p700 CHLOROPHYLL A APOPROTEIN APS I gene; PSII: Photosystem II; PSII: PHOTOSYSTEM II Q(b)gene; ROS: Reactive oxygen species; SBD: SPL binding domain; SPL: SQUAMOSA-PROMOTER BINDING PROTEIN-LIKE; WT: Wild-type

\section{Acknowledgments}

The authors acknowledge Dr. Justin Renaud for his help with LCMS and GCMS.

\section{Authors' contributions}

BAF and MA developed materials; BAF performed the experiments; SEK and $\mathrm{AH}$ supervised the project; $\mathrm{BAF}$ and $\mathrm{AH}$ designed the research; $\mathrm{BAF}, \mathrm{MA}$, MYG, SEK and AH wrote, revised and approved the manuscript.

\section{Funding}

The research is funded through grants from Agriculture and Agri-Food Canada and the Natural Science and Engineering Research Council of Canada to $\mathrm{AH}$. The funding agencies had no role in the design of the study; collection, analysis, and interpretation of data; and in writing the manuscript.

\section{Availability of data and materials}

Data used in this study are provided as 'additional file.xIsx' as a supplementary file.

\section{Ethics approval and consent to participate} Not applicable.

\section{Consent for publication}

These requirements are not applicable to the current manuscript.

\section{Competing interests}

The authors declare that they have no competing interests.

\section{Author details}

${ }^{1}$ Agriculture and Agri-Food Canada, 1391 Sandford Street, London, Ontario N5V 4T3, Canada. '2Department of Biology, University of Western Ontario, 1151 Richmond Street, London, Ontario N6A4B7, Canada. ${ }^{3}$ Center of Agricultural Biochemistry and Biotechnology, University of Agriculture, Faisalabad, Pakistan. ${ }^{4}$ Agriculture and Agri-Food Canada, 107 Science Place, Saskatoon, Saskatchewan S7N OX2 (retired), Canada.

Received: 16 April 2019 Accepted: 27 September 2019

Published online: 21 October 2019

\section{References}

1. Ray DK, Gerber JS, MacDonald GK, West PC. Climate variation explains a third of global crop yield variability. Nat Commun. 2015;6:5989.

2. Olesen JE, Trnka M, Kersebaum KC, Skjelvåg AO, Seguin B, Peltonen-Sainio $P$, et al. Impacts and adaptation of European crop production systems to climate change. Eur J Agron. 2011;34(2):96-112.

3. Mba C, Guimaraes EP, Ghosh K. Re-orienting crop improvement for the changing climatic conditions of the 21st century. Agric Food Secur. 2012; 1(1):1-17.
4. Zhou M, Luo H. MicroRNA-mediated gene regulation: potential applications for plant genetic engineering. Plant Mol Biol. 2013;83(1):59-75.

5. Sun G. MicroRNAs and their diverse functions in plants. Plant Mol Biol. 2012; 80(1):17-36.

6. Zhang BH, Pan XP, Cannon CH, Cobb GP, Anderson TA. Identification and characterization of new plant microRNAs using EST analysis. Cell Res. 2005; 15:336-60.

7. Aung B, Gruber MY, Amyot L, Omari K, Bertrand A, Hannoufa A. Ectopic expression of LjmiR156 delays flowering, enhances shoot branching, and improves forage quality in alfalfa. Plant Biotechnol Rep. 2015a;9(6):379-93.

8. Gao R, Austin RS, Amyot L, Hannoufa A. Comparative transcriptome investigation of global gene expression changes caused by miR156 overexpression in Medicago sativa. BMC Genomics. 2016;17(1):658.

9. Aung B, Gruber MY, Hannoufa A. The MicroRNA156 system: a tool in plant biotechnology. Biocatalysis Agric Biotechnol. 2015c;4(4):432-42.

10. Xie Z, Allen E, Fahlgren N, Calamar A, Givan SA, Carrington JC. Expression of Arabidopsis MIRNA genes. Plant Physiol. 2005;138(4):2145-54.

11. Yamaguchi A, Wu M-F, Yang L, Wu G, Poethig RS, Wagner D. The MicroRNAregulated SBP-box transcription factor SPL3 is a direct upstream activator of LEAFY, FRUITFULL, and APETALA1. Dev Cell. 2009;17(2):268-78.

12. Wei S, Gruber MY, Yu B, Gao M-J, Khachatourians GG, Hegedus DD, et al. Arabidopsis mutant sk156 reveals complex regulation of SPL15 in a miR156controlled gene network. BMC Plant Biol. 2012;12(1):169.

13. Aung B, Gruber MY, Amyot L, Omari K, Bertrand A, Hannoufa A. MicroRNA156 as a promising tool for alfalfa improvement. Plant Biotechnol J. 2015b;13(6):779-90.

14. Xu M, Hu T, Zhao J, Park M-Y, Earley KW, Wu G, et al. Developmental functions of miR156-regulated SQUAMOSA PROMOTER BINDING PROTEIN-LIKE (SPL) genes in Arabidopsis thaliana. PLoS Genet. 2016;12(8):e1006263.

15. Wu G, Park MY, Conway SR, Wang J-W, Weigel D, Poethig RS. The sequential action of miR156 and miR172 regulates developmental timing in Arabidopsis. Cell. 2009:138(4):750-9.

16. Wang L, Sun S, Jin J, Fu D, Yang X, Weng $X$, et al. Coordinated regulation of vegetative and reproductive branching in rice. Proc Natl Acad Sci U S A. 2015;112(50):15504-9.

17. Malik Al, Colmer TD, Lambers H, Setter TL, Schortemeyer M. Short-term waterlogging has long-term effects on the growth and physiology of wheat. New Phytol. 2002:153(2):225-36.

18. Serraj R. Effects of drought stress on legume symbiotic nitrogen fixation: physiological mechanisms. Indian J Exp Biol. 2003:41:1136-41.

19. Matthews C, Arshad M, Hannoufa A. Alfalfa response to heat stress is modulated by microRNA156. Physiol Plant. 2019;165(4):830-42.

20. Arshad M, Gruber MY, Wall K, Hannoufa A. An Insight into microRNA156 role in salinity stress responses of Alfalfa. Front Plant Sci. 2017a;8:356.

21. Arshad M, Feyissa B, Amyot L, Aung B, Hannoufa A. MicroRNA156 improves drought stress tolerance in alfalfa (Medicago sativa) by silencing SPL13. Plant Sci. 2017b;258:122-36.

22. Stief A, Altmann S, Hoffmann K, Pant BD, Scheible WR, Baurle I. Arabidopsis miR156 regulates tolerance to recurring environmental stress through SPL transcription factors. Plant Cell. 2014;26(4):1792-807.

23. Cui L-G, Shan J-X, Shi M, Gao J-P, Lin H-X. The miR156-SPL9-DFR pathway coordinates the relationship between development and abiotic stress tolerance in plants. Plant J. 2014;80(6):1108-17.

24. Pang Y, Wenger JP, Saathoff K, Peel GJ, Wen J, Huhman D, et al. A WD40 repeat protein from Medicago truncatula is necessary for tissue-specific anthocyanin and Proanthocyanidin biosynthesis but not for Trichome development. Plant Physiol. 2009:151(3):1114-29.

25. Verdier J, Zhao J, Torres-Jerez I, Ge S, Liu C, He X, et al. MtPAR MYB transcription factor acts as an on switch for proanthocyanidin biosynthesis in Medicago truncatula. Proc Natl Acad Sci. 2012;109(5):1766-71.

26. Nakabayashi R, Yonekura-Sakakibara K, Urano K, Suzuki M, Yamada $Y$, Nishizawa $T$, et al. Enhancement of oxidative and drought tolerance in Arabidopsis by overaccumulation of antioxidant flavonoids. Plant J. 2014; 77(3):367-79.

27. Degu A, Ayenew B, Cramer GR, Fait A. Polyphenolic responses of grapevine berries to light, temperature, oxidative stress, abscisic acid and jasmonic acid show specific developmental-dependent degrees of metabolic resilience to perturbation. Food Chem. 2016:212:828-36.

28. Ayenew B, Degu A, Manela N, Perl A, Shamir MO, Fait A. Metabolite profiling and transcript analysis reveal specificities in the response of a berry derived cell culture to abiotic stresses. Front Plant Sci. 2015;6:728. 
29. Fait A, Fromm H, Walter D, Galili G, Fernie AR. Highway or byway: the metabolic role of the GABA shunt in plants. Trends Plant Sci. 2008;13(1): $14-9$

30. Argyrokastritis IG, Papastylianou PT, Alexandris S. Leaf water potential and crop water stress index variation for full and deficit irrigated cotton in mediterranean conditions. Agric Agric Sci Procedia. 2015;4:463-70.

31. Cohen M, Goldhamer DA, Fereres E, Girona J, Mata M. Assessment of peach tree responses to irrigation water deficits by continuous monitoring of trunk diameter changes. J Hortic Sci Biotechnol. 2001;76(1):55-60.

32. Goldhamer DA, Fereres E. Irrigation scheduling protocols using continuously recorded trunk diameter measurements. Irrig Sci. 2001;20(3):115-25.

33. Intrigliolo DS, Castel JR. Continuous measurement of plant and soil water status for irrigation scheduling in plum. Irrig Sci. 2004;23(2):93-102.

34. Pinheiro C, Chaves MM. Photosynthesis and drought: can we make metabolic connections from available data? J Exp Bot. 2011;62(3):869-82.

35. Matsuda F. Regular expressions of MS/MS spectra for partial annotation of metabolite features. Metabolomics. 2016;12(7):113.

36. Alonso A, Marsal S, Julià A. Analytical methods in untargeted metabolomics: state of the art in 2015. Front Bioeng Biotechnol. 2015;3:23.

37. Smirnoff $N$. The role of active oxygen in the response of plants to water deficit and desiccation. New Phytol. 1993;125(1):27-58.

38. Cruz de Carvalho MH. Drought stress and reactive oxygen species: production, scavenging and signaling. Plant Signal Behav. 2008;3(3):156-65.

39. Zhang Y, Butelli E, De Stefano R, Schoonbeek H-j, Magusin A, Pagliarani C, et al. Anthocyanins double the shelf life of tomatoes by delaying overripening and reducing susceptibility to gray mold. Curr Biol. 2013; 23(12):1094-100

40. Zhang Y, De Stefano R, Robine M, Butelli E, Bulling K, Hill L, et al. Different reactive oxygen species scavenging properties of flavonoids determine their abilities to extend the shelf life of tomato. Plant Physiol. 2015;169(3):1568-83.

41. Tamura H, Hayashi Y, Sugisawa H, Kondo T. Structure determination of acylated anthocyanins in Muscat bailey a grapes by homonuclear Hartmann-Hahn (HOHAHA) spectroscopy and liquid chromatography-mass spectrometry. Phytochem Anal. 1994;5(4):190-6.

42. He F, Mu L, Yan G-L, Liang N-N, Pan Q-H, Wang J, et al. Biosynthesis of anthocyanins and their regulation in colored grapes. Molecules. 2010; 15(12):9057.

43. Hochberg U, Degu A, Toubiana D, Gendler T, Nikoloski Z, Rachmilevitch S, et al. Metabolite profiling and network analysis reveal coordinated changes in grapevine water stress response. BMC Plant Biol. 2013;13(1):184.

44. Huan L, Xie X, Zheng Z, Sun F, Wu S, Li M, et al. Positive correlation between PSI response and oxidative pentose phosphate pathway activity during salt stress in an intertidal macroalga. Plant Cell Physiol. 2014;55(8): 1395-403.

45. Stincone A, Prigione A, Cramer T, Wamelink MMC, Campbell K, Cheung E, et al. The return of metabolism: biochemistry and physiology of the pentose phosphate pathway. Biol Rev Camb Philos Soc. 2015;90(3):927-63.

46. Lotkowska ME, Tohge T, Fernie AR, Xue G-P, Balazadeh S, Mueller-Roeber B. The Arabidopsis transcription factor MYB112 promotes anthocyanin formation during salinity and under high light stress. Plant Physiol. 2015; 169(3):1862-80.

47. Schulz P, Neukermans J, Van Der Kelen K, Mühlenbock P, Van Breusegem F, Noctor G, et al. Chemical PARP inhibition enhances growth of Arabidopsis and reduces anthocyanin accumulation and the activation of stress protective mechanisms. PLoS One. 2012;7(5):e37287.

48. Gould KS, Dudle DA, Neufeld HS. Why some stems are red: cauline anthocyanins shield photosystem II against high light stress. J Exp Bot. 2010; 61(10):2707-17.

49. Nozzolillo C, McNeill J. Anthocyanin pigmentation in seedlings of selected species of Phaseolus and Vigna (Fabaceae). Can J Bot. 1985;63(6):1066-71.

50. Abe H, Urao T, Ito T, Seki M, Shinozaki K, Yamaguchi-Shinozaki K. Arabidopsis AtMYC2 (bHLH) and AtMYB2 (MYB) function as transcriptional activators in abscisic acid signaling. Plant Cell. 2003;15(1):63-78.

51. Tuteja N. Abscisic acid and abiotic stress signaling. Plant Signal Behav. 2007; 2(3):135-8.

52. Gao R, Gruber MY, Amyot L, Hannoufa A. SPL13 regulates shoot branching and flowering time in Medicago sativa. Plant Mol Biol. 2017:96(1-2):119-33.

53. Gao Y, Liu J, Chen Y, Tang H, Wang Y, He Y, et al. Tomato SIAN11 regulates flavonoid biosynthesis and seed dormancy by interaction with bHLH proteins but not with MYB proteins. Horticulture Res. 2018;5(1):27.
54. Mpelasoka F, Hennessy K, Jones R, Bates B. Comparison of suitable drought indices for climate change impacts assessment over Australia towards resource management. Int J Climatol. 2008;28(10):1283-92.

55. Obidiegwu JE, Bryan GJ, Jones HG, Prashar A. Coping with drought: stress and adaptive responses in potato and perspectives for improvement. Front Plant Sci. 2015;6:542.

56. Kayum MA, Kim H-T, Nath UK, Park J-I, Kho KH, Cho Y-G, et al. Research on biotic and abiotic stress related genes exploration and prediction in Brassica rapa and B. oleracea: a review. Plant Breed Biotechnol. 2016;4(2):135-44.

57. Pandey $V$, Shukla A. Improving crop yield under drought stress through physiological breeding. In: Hossain MA, Wani SH, Bhattacharjee S, Burritt DJ, Tran L-SP, editors. Drought Stress Tolerance in Plants, Vol 1. Switzerland: Physiology and Biochemistry: Springer International Publishing; 2016. p. 331-48.

58. Chen $\mathrm{H}, \mathrm{Li} \mathrm{Z}$, Xiong L. A plant microRNA regulates the adaptation of roots to drought stress. FEBS Lett. 2012;586(12):1742-7.

59. Xiong L, Wang R-G, Mao G, Koczan JM. Identification of drought tolerance determinants by genetic analysis of root response to drought stress and abscisic acid. Plant Physiol. 2006;142(3):1065-74.

60. Liang Y, Mitchell DM, Harris JM. Abscisic acid rescues the root meristem defects of the Medicago truncatula latd mutant. Dev Biol. 2007;304(1): 297-307.

61. Gao R, Wang Y, Gruber MY, Hannoufa A. miR156/SPL10 Modulates Lateral Root Development, Branching and Leaf Morphology in Arabidopsis by Silencing AGAMOUS-LIKE 79. Front Plant Sci. 2018;8:2226.

62. Aranjuelo I, Molero G, Erice G, Avice JC, Nogués S. Plant physiology and proteomics reveals the leaf response to drought in alfalfa (Medicago sativa L.). J Exp Bot. 2011:62(1):111-23.

63. Flagella Z, Pastore D, Campanile RG, Fonzo ND. The quantum yield of photosynthetic electron transport evaluated by chlorophyll fluorescence as an indicator of drought tolerance in durum wheat. J Agric Sci. 1995;125(3):325-9.

64. Sharma DK, Andersen SB, Ottosen C-O, Rosenqvist E. Wheat cultivars selected for high Fv/Fm under heat stress maintain high photosynthesis, total chlorophyll, stomatal conductance, transpiration and dry matter. Physiol Plant. 2015;153(2):284-98.

65. Gautam A, Agrawal D, SaiPrasad SV, Jajoo A. A quick method to screen high and low yielding wheat cultivars exposed to high temperature. Physiol Mol Biol Plants. 2014;20(4):533-7.

66. Murchie EH, Lawson T. Chlorophyll fluorescence analysis: a guide to good practice and understanding some new applications. J Exp Bot. 2013;64(13):3983-98.

67. Su L, Dai Z, Li S, Xin H. A novel system for evaluating drought-cold tolerance of grapevines using chlorophyll fluorescence. BMC Plant Biol. 2015;15(1):82

68. Merchuk-Ovnat L, Fahima T, Krugman T, Saranga Y. Ancestral QTL alleles from wild emmer wheat improve grain yield, biomass and photosynthesis across environments in modern wheat. Plant Sci. 2016;251:23-34.

69. Mishra KB, lannacone R, Petrozza A, Mishra A, Armentano N, La Vecchia G, et al. Engineered drought tolerance in tomato plants is reflected in chlorophyll fluorescence emission. Plant Sci. 2012;182:79-86.

70. Cramer GR, Urano K, Delrot S, Pezzotti M, Shinozaki K. Effects of abiotic stress on plants: a systems biology perspective. BMC Plant Biol. 2011;11(1):163.

71. Hochberg U, Degu A, Cramer GR, Rachmilevitch S, Fait A. Cultivar specific metabolic changes in grapevines berry skins in relation to deficit irrigation and hydraulic behavior. Plant Physiol Biochem. 2015;88:42-52.

72. Batushansky A, Kirma M, Grillich N, Pham PA, Rentsch D, Galili G, et al. The transporter GAT1 plays an important role in GABA-mediated carbonnitrogen interactions in Arabidopsis. Front Plant Sci. 2015;6(September):785.

73. Naya L, Ladrera R, Ramos J, González EM, Arrese-lgor C, Minchin FR, et al. The response of carbon metabolism and antioxidant defenses of alfalfa nodules to drought stress and to the subsequent recovery of plants. Plant Physiol. 2007;144(2):1104-14.

74. Larrainzar E, Wienkoop S, Scherling C, Kempa S, Ladrera R, Arrese-lgor C, et al. Carbon metabolism and bacteroid functioning are involved in the regulation of nitrogen fixation in Medicago truncatula under drought and recovery. Mol Plant-Microbe Interact. 2009;22(12):1565-76.

75. Renault H, Roussel V, El Amrani A, Arzel M, Renault D, Bouchereau A, et al. The Arabidopsis pop2-1 mutant reveals the involvement of GABA transaminase in salt stress tolerance. BMC Plant Biol. 2010;10:20.

76. Vijayakumari K, Puthur JT. $Y$-Aminobutyric acid (GABA) priming enhances the osmotic stress tolerance in Piper nigrum Linn. Plants subjected to PEGinduced stress. Plant Growth Regul. 2016;78(1):57-67. 
77. Li Z, Yu J, Peng Y, Huang B. Metabolic pathways regulated by Yaminobutyric acid (GABA) contributing to heat tolerance in creeping bentgrass (Agrostis stolonifera). Sci Rep. 2016;6:30338.

78. Aranjuelo I, Tcherkez G, Molero G, Gilard F, Avice J-C, Nogués S. Concerted changes in $\mathrm{N}$ and $\mathrm{C}$ primary metabolism in alfalfa (Medicago sativa) under water restriction. J Exp Bot. 2013;64(4):885-97.

79. Ray H, Yu M, Auser P, Blahut-Beatty L, McKersie B, Bowley S, et al. Expression of anthocyanins and proanthocyanidins after transformation of alfalfa with maize Lc. Plant Physiol. 2003;132(3):1448-63.

80. Dixon RA, Sumner LW. Legume natural products: understanding and manipulating complex pathways for human and animal health. Plant Physiol. 2003;131(3):878-85

81. McMahon LR, McAllister TA, Berg BP, Majak W, Acharya SN, Popp JD, et al. A review of the effects of forage condensed tannins on ruminal fermentation and bloat in grazing cattle. Can J Plant Sci. 2000;80(3):469-85.

82. Aerts RJ, Barry TN, McNabb WC. Polyphenols and agriculture: beneficial effects of proanthocyanidins in forages. Agric Ecosyst Environ. 1999;75(1-2):1-12.

83. Li H, Qiu J, Chen F, Lv X, Fu C, Zhao D, et al. Molecular characterization and expression analysis of DIHYDROFLAVONOL 4-REDUCTASE (DFR) gene in Saussurea medusa. Mol Biol Rep. 2012;39(3):2991-9.

84. Wang Z, Wang Y, Kohalmi SE, Amyot L, Hannoufa A. SQUAMOSA PROMOTER BINDING PROTEIN-LIKE 2 controls floral organ development and plant fertility by activating ASYMMETRIC LEAVES 2 in Arabidopsis thaliana. Plant Mol Biol. 2016;92(6):661-74.

85. Yu N, Cai WJ, Wang S, Shan CM, Wang LJ, Chen XY. Temporal control of trichome distribution by microRNA156-targeted SPL genes in Arabidopsis thaliana. Plant Cell. 2010;22(7):2322-35.

86. Gou J-Y, Felippes FF, Liu C-J, Weigel D, Wang J-W. Negative regulation of anthocyanin biosynthesis in Arabidopsis by a miR156-targeted SPL transcription factor. Plant Cell. 2011;23(4):1512-22.

87. Badhan A, Jin L, Wang Y, Han S, Kowalczys K, Brown DC, et al. Expression of a fungal ferulic acid esterase in alfalfa modifies cell wall digestibility. Biotechnol Biofuels. 2014:7(1):39.

88. Pluskal T, Castillo S, Villar-Briones A, Orešič M. MZmine 2: modular framework for processing, visualizing, and analyzing mass spectrometrybased molecular profile data. BMC Bioinformatics. 2010;11(1):395.

89. Cheok CY, Chin NL, Yusof YA, Talib RA, Law CL. Optimization of total monomeric anthocyanin (TMA) and total phenolic content (TPC) extractions from mangosteen (Garcinia mangostana Linn.) hull using ultrasonic treatments. Ind Crop Prod. 2013;50:1-7.

90. Lee J, Durst R, Wrolstad R. Determination of total monomeric anthocyanin pigment content of fruit juices, beverages, natural colorants, and wines by the pH differential method: collaborative study. J AOAC Int. 2005;88(5): 1269-78.

91. Duursma RA. Plantecophys - an R package for analysing and modelling leaf gas exchange data. PLoS One. 2015;10(11):e0143346.

92. Sawada Y, Nakabayashi R, Yamada Y, Suzuki M, Sato M, Sakata A, et al. RIKEN tandem mass spectral database (ReSpect) for phytochemicals: a plantspecific MS/MS-based data resource and database. Phytochemistry. 2012;82: 38-45.

93. Gendrel A-V, Lippman Z, Martienssen R, Colot V. Profiling histone modification patterns in plants using genomic tiling microarrays. Nat Methods. 2005;2(3):213-8.

\section{Publisher's Note}

Springer Nature remains neutral with regard to jurisdictional claims in published maps and institutional affiliations.

Ready to submit your research? Choose BMC and benefit from:
- fast, convenient online submission
- thorough peer review by experienced researchers in your field
- rapid publication on acceptance
- support for research data, including large and complex data types
- gold Open Access which fosters wider collaboration and increased citations
- maximum visibility for your research: over 100M website views per year
At BMC, research is always in progress.
Learn more biomedcentral.com/submissions

